\title{
Quasiperiodic Solutions of Completely Resonant Wave Equations with Quasiperiodic Forced Terms
}

\author{
Yixian Gao, ${ }^{1,2}$ Weipeng Zhang, ${ }^{1}$ and Jing Chang ${ }^{3}$ \\ ${ }^{1}$ School of Mathematics and Statistics, Northeast Normal University, Changchun, Jilin 130024, China \\ ${ }^{2}$ Key Laboratory of Symbolic Computation and Knowledge Engineering, Ministry of Education, Jilin University, Changchun, \\ Jilin 130012, China \\ ${ }^{3}$ Fundamental Department, Aviation University of Air Force, Changchun 130023, China
}

Correspondence should be addressed to Weipeng Zhang; wpzhang808@163.com

Received 28 February 2014; Revised 25 April 2014; Accepted 28 April 2014; Published 18 May 2014

Academic Editor: Yongli Song

Copyright (C) 2014 Yixian Gao et al. This is an open access article distributed under the Creative Commons Attribution License, which permits unrestricted use, distribution, and reproduction in any medium, provided the original work is properly cited.

This paper is concerned with the existence of quasiperiodic solutions with two frequencies of completely resonant, quasiperiodically forced nonlinear wave equations subject to periodic spatial boundary conditions. The solutions turn out to be, at the first order, the superposition of traveling waves, traveling in the opposite or the same directions. The proofs are based on the variational Lyapunov-Schmidt reduction and the linking theorem, while the bifurcation equations are solved by variational methods.

\section{Introduction}

This paper is devoted to the study of the existence of smallamplitude quasiperiodic solutions of completely resonant forced nonlinear wave equations like

$$
u_{t t}-u_{x x}+f\left(\omega_{1} t, \omega_{2} t, x, u\right)=0,
$$

with periodic boundary conditions

$$
u(t, x)=u(t, x+2 \pi), \quad(t, x) \in \mathbb{R}^{2},
$$

while the nonlinear forced term $f$ is

$$
f\left(\omega_{1} t, \omega_{2} t, x, u\right):=\sum_{k \geq 3} a_{k}\left(\omega_{1} t+x, \omega_{2} t+x\right) u^{k},
$$

when the traveling waves are in the same directions, and $f$ is

$$
f\left(\omega_{1} t, \omega_{2} t, x, u\right):=\sum_{k \geq 3} a_{k}\left(\omega_{1} t-x, \omega_{2} t+x\right) u^{k}
$$

when the traveling waves are in the opposite directions. Moreover, the nonlinear forced terms are all analytic in a neighborhood of $u=0$.
Periodic or quasiperiodic solutions in nonresonant PDEs have been obtained, for instance, in [1-12] by the LyapunovSchmidt reduction together with Nash-Moser theory and KAM theory, while the completely resonant autonomous PDEs have been originally studied by variational methods starting from Rabinowitz [13-19]. They obtained the existence of periodic solutions with period being a rational multiple of $\pi$, and such solutions correspond to a zero-measure set of values of the amplitudes. The case with period being irrational of $\pi$, which in principle could provide a large measure of values, has been mostly studied under strong Diophantine conditions; see [20-25] and the references therein. In $[26,27]$, using the Lindstedt series method, Gentile and Procesi obtained the existence of periodic solutions for a large measure set of frequencies for the nonlinear wave equations and nonlinear Schrödinger equations with periodic boundary conditions. In [28], Yuan obtained the existence of quasiperiodic solution for a large measure set of at least three dimensional rotation vectors by the KAM method. In [29], under the periodic boundary condition and with the periodic forced nonlinearities $f\left(\omega_{1} t, u\right)=a\left(\omega_{1} t\right) u^{2 d-1}+$ $O\left(u^{2 d}\right), d>2$, Berti and Procesi got the existence of quasiperiodic solution of nonlinear wave equation in the form of $v(t, x)=u\left(\omega_{1} t, \omega_{2} t+x\right)$. In [30], Procesi firstly 
obtained the quasiperiodic solutions with two frequencies in the form of $v(t, x)=u\left(\omega_{1} t+x, \omega_{2} t-x\right)$ for the specific nonlinearities $f=u^{3}+O\left(u^{5}\right)$, where the forced terms do not depend on the time and the bifurcation equations are solved by ODE methods. In [31], Baldi proved the existence of small-amplitude quasiperiodic solutions in the form of $v(t, x)=u\left(\omega_{1} t+x, \omega_{2} t+x\right)$ with the general nonlinearities $f=u^{3}+O\left(u^{4}\right)$, which also do not depend on the time. In [32], they considered the existence of quasiperiodic solution of the forced wave equation, in which the solutions are traveling in opposite directions. However, they did not give the regularity of the solutions, and the results are the special case of our results in Section 4. Moreover, we mention the work [33] of Bambusi, where a simple proof of an infinite-dimensional extension of the Lyapunov center theorem is given.

In this paper, for the completely resonant wave equation (1) subjecting to the quasiperiodic forced terms, we will prove the existence and regularity of quasiperiodic solutions with two frequencies, $\omega_{1}, \omega_{2}$, in both of the following two cases.

Case (A1). The first case considers the wave traveling in the same directions $u(t, x)=v\left(\omega_{1} t+x, \omega_{2} t+x\right)$.

Case (A2). The second case considers the wave traveling in opposite directions $u(t, x)=v\left(\omega_{1} t-x, \omega_{2} t+x\right)$.

\section{Main Results}

We look for quasiperiodic solutions $u(t, x)$ of (1) of the following form:

(in the same directions)

$$
\begin{gathered}
u(t, x)=v\left(\omega_{1} t+x, \omega_{2} t+x\right)=v\left(\varphi_{1}, \varphi_{2}\right), \\
v\left(\varphi_{1}, \varphi_{2}\right)=v\left(\varphi_{1}+2 k_{1} \pi, \varphi_{2}+2 k_{2} \pi\right), \quad \forall k_{1}, k_{2} \in \mathbb{Z},
\end{gathered}
$$

with frequencies $\omega=\left(\omega_{1}, \omega_{2}\right)=\left(1+\epsilon, 1+a \epsilon^{2}\right), a \in \mathbb{R}^{-}$, or (in opposite directions)

$$
\begin{gathered}
u(t, x)=v\left(\omega_{1} t-x, \omega_{2} t+x\right)=v\left(\varphi_{1}, \varphi_{2}\right), \\
v\left(\varphi_{1}, \varphi_{2}\right)=v\left(\varphi_{1}+2 k_{1} \pi, \varphi_{2}+2 k_{2} \pi\right), \quad \forall k_{1}, k_{2} \in \mathbb{Z},
\end{gathered}
$$

with frequencies $\omega=\left(\omega_{1}, \omega_{2}\right)=(1+\epsilon, 1+a \epsilon), a \in \mathbb{R}^{-}$, imposing the frequencies $\omega=\left(\omega_{1}, \omega_{2}\right)$ to be close to linear frequency 1 . Therefore, finding the quasiperiodic solutions of (1) with frequencies, respectively, $\left(\omega_{1}, \omega_{2}\right)$ is equivalent to finding $2 \pi$ periodic solutions with respect to $\left(\varphi_{1}, \varphi_{2}\right)$ for the following equations:

(in the same directions)

$$
\begin{aligned}
\left(\partial_{t t}^{2}-\partial_{x x}^{2}\right) v & =\left(\partial_{t}-\partial_{x}\right) \circ\left(\partial_{t}+\partial_{x}\right) v \\
& =\left[\omega_{1} \partial_{\varphi_{1}}+\omega_{2} \partial_{\varphi_{2}}-\partial_{\varphi_{1}}-\partial_{\varphi_{2}}\right]
\end{aligned}
$$

$$
\begin{aligned}
& \circ\left[\omega_{1} \partial_{\varphi_{1}}+\omega_{2} \partial_{\varphi_{2}}+\partial_{\varphi_{1}}+\partial_{\varphi_{2}}\right] v \\
= & \left(\left(\omega_{1}^{2}-1\right) \partial_{\varphi_{1}}^{2}+\left(\omega_{2}^{2}-1\right) \partial_{\varphi_{2}}^{2}\right. \\
& \left.+2\left(\omega_{1} \omega_{2}-1\right) \partial_{\varphi_{1}} \partial_{\varphi_{2}}\right) v \\
= & -f\left(\varphi_{1}, \varphi_{2}, v\right),
\end{aligned}
$$

(in opposite directions)

$$
\begin{aligned}
\left(\partial_{t t}^{2}-\partial_{x x}^{2}\right) v= & \left(\partial_{t}-\partial_{x}\right) \circ\left(\partial_{t}+\partial_{x}\right) v \\
= & {\left[\omega_{1} \partial_{\varphi_{1}}+\omega_{2} \partial_{\varphi_{2}}+\partial_{\varphi_{1}}-\partial_{\varphi_{2}}\right] } \\
& \circ\left[\omega_{1} \partial_{\varphi_{1}}+\omega_{2} \partial_{\varphi_{2}}-\partial_{\varphi_{1}}+\partial_{\varphi_{2}}\right] v \\
= & \left(\left(\omega_{1}^{2}-1\right) \partial_{\varphi_{1}}^{2}+\left(\omega_{2}^{2}-1\right) \partial_{\varphi_{2}}^{2}\right. \\
& \left.+2\left(\omega_{1} \omega_{2}+1\right) \partial_{\varphi_{1}} \partial_{\varphi_{2}}\right) v \\
= & -f\left(\varphi_{1}, \varphi_{2}, v\right) .
\end{aligned}
$$

We assume that the quasiperiodic forced term $f: \mathbb{T}^{2} \times \mathbb{R} \rightarrow$ $\mathbb{R}$,

$$
f\left(\varphi_{1}, \varphi_{2}, v\right)=a_{3}\left(\varphi_{1}, \varphi_{2}\right) v^{3}+O\left(v^{4}\right)
$$

is analytic in $v$ but has only finite regularity in $\varphi_{1}, \varphi_{2}$. More precisely,

(H) $f\left(\varphi_{1}, \varphi_{2}, v\right):=\sum_{k=3}^{\infty} a_{k}\left(\varphi_{1}, \varphi_{2}\right) v^{k}$, and the coefficients $a_{k}\left(\varphi_{1}, \varphi_{2}\right) \in H^{1}\left(\mathbb{T}^{2}\right)$ verify, for some $r>0$, $\sum_{k=3}^{\infty}\left|a_{k}\right|_{H^{1}} r^{k}<\infty$. The function $f\left(\varphi_{1}, \varphi_{2}, v\right)$ is not identically constant in $\left(\varphi_{1}, \varphi_{2}\right)$.

We look for solutions $v$ of (7)-(8) in the Banach space

$$
\begin{aligned}
& \mathscr{H}_{\sigma, s} \\
& :=\left\{v(\varphi)=\sum_{\left(l_{1}, l_{2}\right) \in \mathbb{Z}^{2}} \widehat{v}_{\left(l_{1}, l_{2}\right)} e^{i l_{1} \varphi_{1}} e^{i l_{2} \varphi_{2}}: \widehat{v}_{\left(l_{1}, l_{2}\right)}^{*}=\widehat{v}_{\left(-l_{1},-l_{2}\right)},\right. \\
& \left.|v|_{\sigma, s}=\sum_{\left(l_{1}, l_{2}\right) \in \mathbb{Z}^{2}}\left|\widehat{v}_{\left(l_{1}, l_{2}\right)}\right| e^{\left|l_{2}\right| \sigma}\left(\max \left\{0,\left|l_{1}\right|\right\}\right)^{s}<+\infty\right\},
\end{aligned}
$$

where $l=\left(l_{1}, l_{2}\right) \in \mathbb{Z}^{2}, \widehat{v}_{\left(l_{1}, l_{2}\right)}^{*}$ denotes its complex conjugate, and $\sigma>0, s \geq 0$.

The space $\mathscr{H}_{\sigma, s}$ is a Banach algebra with respect to multiplications of functions, namely,

$$
v_{1}, v_{2} \in \mathscr{H}_{\sigma, s} \Longrightarrow v_{1} v_{2} \in \mathscr{H}_{\sigma, s}, \quad\left|v_{1} v_{2}\right|_{\sigma, s} \leq C\left|v_{1}\right|_{\sigma, s}\left|v_{2}\right|_{\sigma, s} .
$$

We will prove the following theorems. 
Theorem 1. Assume that the nonlinearity $f$ satisfies $(H)$ and $a_{3}\left(\varphi_{1}, \varphi_{2}\right) \neq 0, \forall\left(\varphi_{1}, \varphi_{2}\right) \in \mathbb{T}^{2}$. Let $\mathscr{B}_{\gamma}$ be the uncountable zero-measure Cantor set

$$
\begin{aligned}
& \mathscr{B}_{\gamma} \\
& :=\left\{(a, \epsilon) \in \mathbb{R}^{-} \times \mathbb{R}, 1+\epsilon \neq 0,1+a \epsilon^{2} \neq 0,2+a \epsilon^{2} \neq 0,\right. \\
& \quad\left(\frac{2+\epsilon}{2+a \epsilon^{2}}, a \epsilon\right) \in \mathscr{C}_{\gamma_{1}} \cap\left(1-\epsilon_{0}, 1+\epsilon_{0}\right) \\
& \left.\quad \times \mathscr{C}_{\gamma_{2}} \cap\left(-\epsilon_{0}, \epsilon_{0}\right), \frac{1+\epsilon}{1+a \epsilon^{2}} \notin \mathbb{Q}, \text { for } \epsilon_{0} \in\left(0, \frac{1}{2}\right)\right\},
\end{aligned}
$$

where $\mathscr{C}_{\gamma_{i}}, i=1,2$, are sets of badly approximate numbers defined as

$$
\begin{aligned}
& \mathscr{C}_{\gamma_{1}}:=\left\{\frac{2+\epsilon}{2+a \epsilon^{2}}:\left|l_{2}+\frac{2+\epsilon}{2+a \epsilon^{2}} l_{1}\right|>\frac{\gamma}{\left|l_{1}\right|}\right\}, \\
& \mathscr{C}_{\gamma_{2}}:=\left\{a \epsilon:\left|l_{1}+a \epsilon l_{2}\right|>\frac{\gamma}{\left|l_{2}\right|}\right\}
\end{aligned}
$$

for $\forall l_{1}, l_{2} \in \mathbb{Z} \backslash\{0\}$, and $0<\gamma<1 / 4$. There exist constants $\bar{\sigma}>0, \bar{s}>2$, and $\bar{\epsilon}(R)>0$, such that, for $(a, \epsilon) \in \mathscr{B}_{\gamma},|\epsilon| / \gamma<$ $\bar{\epsilon}(R)$, there exists a solution $v\left(\epsilon, \varphi_{1}, \varphi_{2}\right) \in \mathscr{H}_{\bar{\sigma}, \bar{s}}$ of $(7)$, having the form

$$
\begin{aligned}
v\left(\epsilon, \varphi_{1}, \varphi_{2}\right) \\
\quad=|\epsilon|\left(\widehat{q}_{0}+\widehat{q}_{-}\left(\varphi_{2}\right)+\widehat{q}_{+}\left(\varphi_{1}+\varphi_{2}\right)+\widehat{p}\left(\epsilon, \varphi_{1}, \varphi_{2}\right)\right),
\end{aligned}
$$

with

$$
\left|\widehat{p}\left(\epsilon, \varphi_{1}, \varphi_{2}\right)\right|_{\bar{\sigma}, \bar{s}} \leq \bar{C}\left(\frac{|\epsilon|}{\gamma}\right),
$$

where $\bar{C}$ is a constant. As a consequence, (1) possesses the quasiperiodic solutions, traveling in the same directions, $u(\epsilon, t, x)=v\left(\epsilon, \omega_{1} t+x, \omega_{2} t+x\right)$, with two frequencies $\left(\omega_{1}, \omega_{2}\right)=\left(1+\epsilon, 1+a \epsilon^{2}\right)$.

Theorem 2. Assume that $f$ satisfies assumption $(H)$ and $a_{3}\left(\varphi_{1}, \varphi_{2}\right) \neq 0, \forall\left(\varphi_{1}, \varphi_{2}\right) \in \mathbb{T}^{2}$. Let $\mathscr{D}_{\gamma} \subset\left(-\epsilon_{0}, \epsilon_{0}\right) \times\left(-\epsilon_{0}, \epsilon_{0}\right)$ be the uncountable zero-measure Cantor set

$$
\begin{aligned}
\mathscr{D}_{\gamma}:= & \left\{(a, \epsilon) \in \mathbb{R}^{-} \times \mathbb{R},\left(\frac{a \epsilon}{2+\epsilon}, \frac{\epsilon}{2+a \epsilon}\right) \in \mathscr{E}_{\gamma},\right. \\
& \left.\frac{1+\epsilon}{1+a \epsilon} \notin \mathbb{Q}, 1+\epsilon \neq 0,1+a \epsilon \neq 0,2+a \epsilon \neq 0\right\},
\end{aligned}
$$

where $\mathscr{E}_{\gamma}$ is a set of badly approximate numbers defined as

$$
\begin{aligned}
& \mathscr{E}_{\gamma} \\
& :=\left\{\left(\frac{a \epsilon}{2+\epsilon}, \frac{\epsilon}{2+a \epsilon}\right):=\left(\epsilon_{1}, \epsilon_{2}\right) \in\left(-\epsilon_{0}, \epsilon_{0}\right) \times\left(-\epsilon_{0}, \epsilon_{0}\right):\right. \\
& \left.\quad\left|l_{1}+\epsilon_{1} l_{2}\right|>\frac{\gamma}{\left|l_{2}\right|},\left|l_{2}+\epsilon_{2} l_{1}\right|>\frac{\gamma}{\left|l_{1}\right|}\right\}
\end{aligned}
$$

for $\forall l_{1}, l_{2} \in \mathbb{Z} \backslash\{0\}$, and $0<\gamma<1 / 4, \epsilon_{0} \in(0,1 / 2)$. There exist positive numbers $\bar{\sigma}, \bar{\epsilon}, \bar{C}, \bar{s}>2$, such that, $\forall(a, \epsilon) \in \mathscr{D}_{\gamma}$, (8) admits solutions in the form of

$$
\begin{aligned}
v_{a, \epsilon}(t, x)= & v\left(\epsilon, \varphi_{1}, \varphi_{2}\right) \\
= & \sqrt{|\epsilon|}\left(\widehat{q}_{0}+\widehat{q}_{-}\left(\varphi_{2}\right)+\widehat{q}_{+}\left(\varphi_{1}\right)\right. \\
& \left.+\widehat{p}\left(\epsilon, \varphi_{1}, \varphi_{2}\right)\right) \in \mathscr{H}_{\bar{\sigma}, s},
\end{aligned}
$$

satisfying

$$
\left|\widehat{p}\left(\epsilon, \varphi_{1}, \varphi_{2}\right)\right|_{\bar{\sigma}, \bar{s}} \leq \bar{C}\left(\frac{|\epsilon|}{\gamma}\right) .
$$

As a consequence, (1) possesses the quasiperiodic solutions, $u_{a, \epsilon}(t, x)=v_{a, \epsilon}((1+\epsilon) t+x,(1+a \epsilon) t-x)$, traveling in opposite directions.

Remark 3. The quasiperiodic solutions of traveling waves we obtained are different from the ones got by KAM methods since the quasiperiodic solutions we get depending on $x$ and $t$ are coupled and in the form of the traveling waves.

Remark 4. We can get the similar result with more general nonlinearity, such as $f\left(\omega_{1} t, \omega_{2} t, x, u\right)=a_{d}\left(\varphi_{1}, \varphi_{2}\right) u^{d}+$ $O\left(u^{(d+1)}\right)$, for any $d \in \mathbb{N}, d \geq 3$.

This paper is organized as follows: we first prove the existence of quasiperiodic solutions, at the first order, to the superposition of traveling waves, traveling in the same directions. In Section 4, we prove the existence of quasiperiodic solutions traveling in opposite directions.

\section{Waves Traveling in the Same Directions}

Substituting $\omega_{1}=1+\epsilon, \omega_{2}=1+a \epsilon^{2}$ into (7), we can obtain the equations

$$
\mathscr{L} v+f\left(\varphi_{1}, \varphi_{2}, v\right)=0,
$$

where, see (7), we have

$$
\begin{aligned}
\mathscr{L}= & \left(\left(\omega_{1}^{2}-1\right) \partial_{\varphi_{1}}^{2}+\left(\omega_{2}^{2}-1\right) \partial_{\varphi_{2}}^{2}+2\left(\omega_{1} \omega_{2}-1\right) \partial_{\varphi_{1}} \partial_{\varphi_{2}}\right) v \\
= & \epsilon\left(2 \partial_{\varphi_{1}}^{2}+2 \partial_{\varphi_{1}} \partial_{\varphi_{2}}\right) \\
& +\epsilon^{2}\left(\partial_{\varphi_{1}}^{2}+\left(2 a+a^{2} \epsilon^{2}\right) \partial_{\varphi_{2}}^{2}+2 a(1+\epsilon) \partial_{\varphi_{1}} \partial_{\varphi_{2}}\right),
\end{aligned}
$$

and $f\left(\varphi_{1}, \varphi_{2}, v\right)=a_{3}\left(\varphi_{1}, \varphi_{2}\right) v^{3}+O\left(v^{4}\right)$. To prove Theorem 1 , instead of looking for solutions of (7) in a shrinking neighborhood of zero, it is convenient to perform the rescaling $v\left(\varphi_{1}, \varphi_{2}\right) \rightarrow \epsilon v\left(\varphi_{1}, \varphi_{2}\right)$, enhancing the relation between the amplitude and the frequencies. Without confusion, we define

$$
\begin{aligned}
\mathscr{L}_{a, \epsilon}= & \left(2 \partial_{\varphi_{1}}^{2}+2 \partial_{\varphi_{1}} \partial_{\varphi_{2}}\right) \\
& +\epsilon\left(\partial_{\varphi_{1}}^{2}+\left(2 a+a^{2} \epsilon^{2}\right) \partial_{\varphi_{2}}^{2}+2 a(1+\epsilon) \partial_{\varphi_{1}} \partial_{\varphi_{2}}\right) \\
= & \mathscr{L}_{0}+\epsilon \mathscr{L}_{1},
\end{aligned}
$$


so the problem becomes

$$
\mathscr{L}_{a, \epsilon}+\epsilon f\left(\varphi_{1}, \varphi_{2}, v, \epsilon\right)=0 .
$$

To find the solutions of (23), we will apply the LyapunovSchmidt reduction method which leads to solving separately a "range equation" and a "bifurcation equation." In order to solve the range equation (avoiding small divisor problems), we restrict $\epsilon$ to the uncountable zero-measure set $\mathscr{B}_{\gamma}$ for Theorem 1, and we apply the Contraction Mapping Theorem; similar nonresonance conditions have been employed, for example, in [21-23, 25, 29, 30].

Equation (23) is the Euler-Lagrange equation of the action functional $\Psi_{\epsilon} \in C^{1}\left(\mathscr{H}_{\sigma, s}, \mathbb{R}\right)$ defined by

$$
\begin{gathered}
\Psi_{\epsilon}(v):=\int_{\mathbb{T}^{2}}\left(\partial_{\varphi_{1}} v\right)^{2}+\left(\partial_{\varphi_{1}} v\right)\left(\partial_{\varphi_{2}} v\right) \\
+\epsilon\left(\frac{1}{2}\left(\partial_{\varphi_{1}} v\right)^{2}+\frac{2 a+a^{2} \epsilon^{2}}{2}\left(\partial_{\varphi_{2}} v\right)^{2}\right. \\
\left.+a(1+\epsilon)\left(\partial_{\varphi_{1}} v\right)\left(\partial_{\varphi_{2}} v\right)\right) \\
-\epsilon F\left(\varphi_{1}, \varphi_{2}, v, \epsilon\right) \\
=\Psi_{0}(v)+\epsilon \Psi_{1}(v, \epsilon),
\end{gathered}
$$

where

$$
\begin{aligned}
F\left(\varphi_{1}, \varphi_{2}, v, \epsilon\right):= & \int_{0}^{v} f\left(\varphi_{1}, \varphi_{2}, \xi, \epsilon\right) d \xi \\
\Psi_{0}(v)= & \int_{\mathbb{T}^{2}}\left(\partial_{\varphi_{1}} v\right)^{2}+\left(\partial_{\varphi_{1}} v\right)\left(\partial_{\varphi_{2}} v\right) \\
\Psi_{1}(v, \epsilon):= & \int_{\mathbb{T}^{2}}\left(\frac{1}{2}\left(\partial_{\varphi_{1}} v\right)^{2}+\frac{2 a+a^{2} \epsilon^{2}}{2}\left(\partial_{\varphi_{2}} v\right)^{2}\right. \\
& \left.+a(1+\epsilon)\left(\partial_{\varphi_{1}} v\right)\left(\partial_{\varphi_{2}} v\right)\right) \\
& -\epsilon F\left(\varphi_{1}, \varphi_{2}, v, \epsilon\right) .
\end{aligned}
$$

To find critical points of $\Psi_{\epsilon}(v)$, we perform a variational Lyapunov-Schmidt reduction inspired by Berti and Bolle [22, 23, 29]; see also Ambrosetti and Badiale [34].

3.1. The Variational Lyapunov-Schmidt Reduction. The operator $\mathscr{L}_{a, \epsilon}$ is diagonal defined on the Banach space $\mathscr{H}_{\sigma, s}$ under the Fourier basis $e_{l}=e^{i l_{1} \varphi_{1}} e^{i l_{2} \varphi_{2}}$ with eigenvalues

$$
\begin{aligned}
-D_{l} & =\left(2 l_{1}^{2}+2 l_{1} l_{2}\right)+\epsilon\left(l_{1}^{2}+\left(2 a+a^{2} \epsilon^{2}\right) l_{2}^{2}+2 a(1+\epsilon) l_{1} l_{2}\right) \\
& =\left(l_{1}+a \epsilon l_{2}\right)\left((2+\epsilon) l_{1}+\left(2+a \epsilon^{2}\right) l_{2}\right) .
\end{aligned}
$$

So, we have

$$
\mathscr{L}_{a, \epsilon}[v]=\sum_{\left(l_{1}, l_{2}\right) \in \mathbb{Z}^{2}} D_{l} \widehat{v}_{l_{1}, l_{2}} e^{i l_{1} \varphi_{1}} e^{i l_{2} \varphi_{2}}, \quad \forall v \in \mathscr{H}_{\sigma, s} .
$$

The critical points of the unperturbed functional $\Psi_{0}$ : $\mathscr{H}_{\sigma, s} \rightarrow \mathbb{R}$ form an infinite-dimensional linear space $Q$, and they are the solutions of the equation

$$
\mathscr{L}_{0} q=\left(2 \partial_{\varphi_{1}}^{2}+2 \partial_{\varphi_{1}} \partial_{\varphi_{2}}\right) q=0
$$

The space $Q$ can be written as

$$
\begin{gathered}
Q=\left\{q=\sum_{\left(l_{1}, l_{2}\right) \in \mathbb{Z}^{2}} \widehat{q}_{l_{1}, l_{2}} e^{i l_{1} \varphi_{1}} e^{i l_{2} \varphi_{2}} \in \mathscr{H}_{\sigma, s} \mid \widehat{q}_{l_{1}, l_{2}}=0,\right. \\
\text { for } \left.l_{1}\left(2 l_{1}+2 l_{2}\right) \neq 0\right\} .
\end{gathered}
$$

In view of the variational argument that we will use to solve the bifurcation equation, we split $Q$ as $Q=Q_{+}+Q_{0}+Q_{-}$, where

$$
\begin{aligned}
Q_{+} & :=\left\{q \in Q: \widehat{q}_{l_{1}, l_{2}}=0, \text { for }\left(l_{1}, l_{2}\right) \notin \Lambda_{+}\right\} \\
& =\left\{q_{+}:=q_{+}(\varphi) \in \mathscr{H}_{\sigma, s}^{0}\right\}, \\
Q_{0} & :=\left\{q: q_{0,0} \in \mathbb{R}\right\}, \\
Q_{-} & :=\left\{q \in Q: \widehat{q}_{l_{1}, l_{2}}=0, \text { for }\left(l_{1}, l_{2}\right) \notin \Lambda_{-}\right\} \\
& =\left\{q_{-}:=q_{-}(\varphi) \in \mathscr{H}_{\sigma, s}^{0}\right\},
\end{aligned}
$$

with

$$
\begin{aligned}
& \Lambda_{+}:=\left\{\left(l_{1}, l_{2}\right) \in \mathbb{Z}^{2}: l_{1}=0, l_{2} \neq 0\right\}, \\
& \Lambda_{-}:=\left\{\left(l_{1}, l_{2}\right) \in \mathbb{Z}^{2}: l_{1}+l_{2}=0,\left(l_{1}, l_{2}\right) \neq(0,0)\right\}, \\
& \Lambda_{0}:=\left\{\left(l_{1}, l_{2}\right) \in \mathbb{Z}^{2}:\left(l_{1}, l_{2}\right) \equiv(0,0)\right\} .
\end{aligned}
$$

We will also use in $Q$ the norm

$$
\begin{aligned}
|q|_{H^{1}}^{2}= & \left|q_{+}\right|_{H^{1}(\mathbb{T})}^{2}+q_{0,0}^{2}+\left|q_{-}\right|_{H^{1}(\mathbb{T})}^{2} \\
& \sim \sum_{\left(l_{1}, l_{2}\right) \in \Lambda_{+} \cup \Lambda_{0} \cup \Lambda_{+}} \widehat{q}_{l_{1}, l_{2}}^{2}\left(\left|l_{1}\right|^{2}+\left|l_{2}\right|^{2}+1\right) .
\end{aligned}
$$

So, we can decompose the space $\mathscr{H}_{\sigma, s}=Q+P$, where

$$
\begin{gathered}
P:=\left\{p=\sum_{l_{1}, l_{2} \in \mathbb{Z}} \widehat{p}_{l_{1}, l_{2}} e^{i l_{1} \varphi_{1}} e^{i l_{2} \varphi_{2}} \in \mathscr{H}_{\sigma, s} \mid \widehat{p}_{l_{1}, l_{2}}=0,\right. \\
\text { for } \left.l_{1}\left(2 l_{1}+2 l_{2}\right)=0\right\} .
\end{gathered}
$$

Projecting (23) onto the closed subspaces $Q$ and $P$, setting $v=q+p \in \mathscr{H}_{\sigma, s}$ with $q \in Q$ and $p \in P$, we obtain

$$
\begin{aligned}
& \text { (Q) } \mathscr{L}_{1}[q]+\Pi_{\mathrm{Q}} f\left(\varphi_{1}, \varphi_{2}, q+p, \epsilon\right)=0, \\
& \text { (P) } \mathscr{L}_{a, \epsilon}[p]+\epsilon \Pi_{P} f\left(\varphi_{1}, \varphi_{2}, q+p, \epsilon\right)=0,
\end{aligned}
$$


where $\Pi_{\mathrm{Q}}: \mathscr{H}_{\sigma, s} \rightarrow \mathrm{Q}, \Pi_{P}: \mathscr{H}_{\sigma, s} \rightarrow P$ are the projectors, respectively, onto $Q$ and $P$.

In order to prove analyticity of the solutions and to highlight the compactness of the problem, we perform a finitedimensional Lyapunov-Schmidt reduction, introducing the decomposition $Q=Q_{1}+Q_{2}$, where

$$
\begin{aligned}
& Q_{1}:=\left\{q=\sum_{\left|l_{1}\right|+\left|l_{2}\right| \leq N} \widehat{q}_{l_{1}, l_{2}} e^{i l_{1} \varphi_{1}} e^{i l_{2} \varphi_{2}} \in Q\right\}, \\
& Q_{2}:=\left\{q=\sum_{\left|l_{1}\right|+\left|l_{2}\right| \leq N} \widehat{q}_{l_{1}, l_{2}} e^{i l_{1} \varphi_{1}} e^{i l_{2} \varphi_{2}} \in Q\right\} .
\end{aligned}
$$

Setting $q=q_{1}+q_{2}$ with $q_{1} \in Q_{1}$ and $q_{2} \in Q_{2}$, we finally get

$$
\begin{gathered}
\left(Q_{1}\right) \mathscr{L}_{1}\left[q_{1}\right]+\Pi_{Q_{1}}\left[f\left(\varphi_{1}, \varphi_{2}, q_{1}+q_{2}+p, \epsilon\right)\right]=0 \\
\Longleftrightarrow d \Psi_{\epsilon}(v)[h]=0, \quad \forall h \in Q_{1} ; \\
\left(Q_{2}\right) \mathscr{L}_{1}\left[q_{2}\right]+\Pi_{Q_{2}}\left[f\left(\varphi_{1}, \varphi_{2}, q_{1}+q_{2}+p, \epsilon\right)\right]=0 \\
\Longleftrightarrow d \Psi_{\epsilon}(v)[h]=0, \quad \forall h \in Q_{2} ; \\
(P) \mathscr{L}_{a, \epsilon}[p]+\epsilon \Pi_{P}\left[f\left(\varphi_{1}, \varphi_{2}, q_{1}+q_{2}+p, \epsilon\right)\right]=0 \\
\Longleftrightarrow d \Psi_{\epsilon}(v)[h]=0, \quad \forall h \in P,
\end{gathered}
$$

where $\Pi_{Q}: \mathscr{H}_{\sigma, s} \rightarrow Q_{i}$ are the projectors onto $Q_{i}(i=1,2)$, and $\Pi_{P}: \mathscr{H}_{\sigma, s} \rightarrow P$ is the projector onto $P$. We will solve first the $\left(Q_{2}\right)-(P)$-equations for all $\left|q_{1}\right|_{H^{1}} \leq 2 R$, provided $\epsilon$ belongs to a suitable Cantor-like set, $|\epsilon| \leq \epsilon_{0}(R)$ is sufficiently small, and $N \geq N_{0}(R)$ is large enough (see Lemma 7). Next, we will solve the $Q_{1}$-equation by means of a variational linking argument; see Section 3.4.

3.2. The $\left(Q_{2}\right)-(P)$-Equations. We first prove that $\mathscr{L}_{a, \epsilon}$ restricted to $P$ has a bounded inverse when $(a, \epsilon)$ belongs to the uncountable zero-measure set

$$
\begin{aligned}
\mathscr{B}_{\gamma} & \\
:= & \left\{(a, \epsilon) \in \mathbb{R}^{-} \times \mathbb{R}, 1+\epsilon \neq 0,1+a \epsilon^{2} \neq 0,2+a \epsilon^{2} \neq 0,\right. \\
& \left(\frac{2+\epsilon}{2+a \epsilon^{2}}, a \epsilon\right) \in \mathscr{C}_{\gamma_{1}} \cap\left(1-\epsilon_{0}, 1+\epsilon_{0}\right) \\
& \left.\times \mathscr{C}_{\gamma_{2}} \cap\left(-\epsilon_{0}, \epsilon_{0}\right), \frac{1+\epsilon}{1+a \epsilon^{2}} \notin \mathbb{Q}, \text { for } \epsilon_{0} \in\left(0, \frac{1}{2}\right)\right\},
\end{aligned}
$$

where $\mathscr{C}_{\gamma_{i}}, i=1,2$, is a set of badly approximate numbers defined by

$$
\begin{aligned}
& \mathscr{C}_{\gamma_{1}}:=\left\{\frac{2+\epsilon}{2+a \epsilon^{2}}:\left|l_{2}+\frac{2+\epsilon}{2+a \epsilon^{2}} l_{1}\right|>\frac{\gamma}{\left|l_{1}\right|}\right\}, \\
& \mathscr{C}_{\gamma_{2}}:=\left\{a \epsilon:\left|l_{1}+a \epsilon l_{2}\right|>\frac{\gamma}{\left|l_{2}\right|}\right\},
\end{aligned}
$$

for $\forall l_{1}, l_{2} \in \mathbb{Z} \backslash\{0\}$, and $0<\gamma<1 / 4 . \mathscr{C}_{\gamma_{i}}, i=1,2$, accumulate at 1 and zero, respectively, from both the right and the left; see $[21,31,35]$.

The operator $\mathscr{L}_{\epsilon}$ is diagonal in the Fourier basis $\left\{e^{i l_{1} \varphi_{1}} e^{i l_{2} \varphi_{2}},\left(l_{1}, l_{2}\right) \in \mathbb{Z}^{2}\right\}$ with eigenvalues

$$
\begin{aligned}
D_{l_{1}, l_{2}} & =-\left(l_{1}+a \epsilon l_{2}\right)\left((2+\epsilon) l_{1}+\left(2+a \epsilon^{2}\right) l_{2}\right) \\
& =-\left(2+a \epsilon^{2}\right)\left(l_{1}+a \epsilon l_{1}\right)\left(l_{2}+\frac{2+\epsilon}{2+a \epsilon^{2}} l_{1}\right) .
\end{aligned}
$$

Lemma 5. For $(a, \epsilon) \in \mathscr{B}_{\gamma}$, the eigenvalues $D_{l_{1}, l_{2}}$ of $\mathscr{L}_{a, \epsilon}$ restricted to $P$ satisfy

$$
\begin{array}{r}
\left|D_{l_{1}, l_{2}}\right|=\left|\left(l_{1}+a \epsilon l_{2}\right)\left((2+\epsilon) l_{1}+\left(2+a \epsilon^{2}\right) l_{2}\right)\right|>\gamma, \\
\forall l_{1}, l_{2} \neq 0 .
\end{array}
$$

As a consequence, the operator $\mathscr{L}_{a, \epsilon}: P \rightarrow P$ has a bounded inverse $\mathscr{L}_{a, \epsilon}^{-1}$ and satisfies

$$
\left|\mathscr{L}_{a, \epsilon}^{-1}[h]\right|_{\sigma, s} \leq \frac{|h|_{\sigma, s}}{\gamma}, \quad \forall h \in P .
$$

Proof. Denote by $[x]$ the nearest integer close to $x$ and by $\{x\}=x-[x]$ its fractional part. If both $l_{1} \neq-\left[a \epsilon l_{2}\right]$ and $l_{2} \neq-\left[\left((2+\epsilon) /\left(2+a \epsilon^{2}\right)\right) l_{1}\right]$, we have

$$
\left|D_{l_{1}, l_{2}}\right|=\left|\left(l_{1}+a \epsilon l_{2}\right)\right| \cdot\left|\left(2+a \epsilon^{2}\right)\right|\left|\left(l_{2}+\frac{2+\epsilon}{2+a \epsilon^{2}} l_{1}\right)\right|>1 .
$$

If $l_{1}=-\left[a \epsilon l_{2}\right]$, then $\left|l_{1}\right|<(1 / 2)\left|l_{2}\right|$, so that $\mid l_{2}+((2+\epsilon) /(2+$ $\left.\left.a \epsilon^{2}\right)\right) l_{1}|>(1 / 2)| l_{2} \mid$. This implies that

$$
\begin{aligned}
& \left|D_{l_{1}, l_{2}}\right| \\
& =\left|l_{1}+a \epsilon l_{2}\right| \cdot\left|2+a \epsilon^{2}\right|\left|\frac{2+\epsilon}{2+a \epsilon^{2}} l_{1}+l_{2}\right| \\
& \geq \frac{\gamma}{\left|l_{2}\right|} \cdot \mid(2+\epsilon) \\
& \times\left(\epsilon\left\{\frac{a \epsilon}{2+\epsilon} l_{2}\right\}+2 l_{2}+a \epsilon\left(1-\frac{\epsilon}{2+\epsilon}\right) l_{2}\right) \mid \geq \gamma .
\end{aligned}
$$

In the same way, if $l_{2}=-\left[\left((2+\epsilon) /\left(2+a \epsilon^{2}\right)\right) l_{1}\right]$, we have

$$
\left|D_{l_{1}, l_{2}}\right| \geq \frac{\gamma}{\left|l_{1}\right|} \cdot\left|\left(2+a \epsilon^{2}\right)\left(\epsilon\left\{\frac{(2+\epsilon) a}{2+a \epsilon^{2}} l_{1}\right\}+\frac{2-2 a \epsilon}{2+a \epsilon^{2}} l_{1}\right)\right| \geq \gamma .
$$

So, the operator $\mathscr{L}_{a, \epsilon}$ restricted to $P$ has a bounded inverse and satisfies

$$
\begin{aligned}
\left|\mathscr{L}_{a, \epsilon}^{-1}[h]\right|_{\sigma, s} & =\sum_{\left(l_{1}, l_{2}\right) \in \mathbb{Z}^{2}} \frac{\left|\widehat{h}_{l_{1}, l_{2}}\right| e^{\left|l_{2}\right| \sigma}\left(\max \left\{0,\left|l_{1}\right|\right\}\right)^{s}}{D_{l_{1}, l_{2}}} \\
& \leq \frac{|h|_{\sigma, s}}{\gamma}, \quad \forall h \in P .
\end{aligned}
$$


Lemma 6. The operator $\mathscr{L}_{1}: Q_{2} \rightarrow Q_{2}$ has a bounded inverse $\mathscr{L}_{1}^{-1}$, satisfying

$$
\left|\mathscr{L}_{1}^{-1}[h]\right|_{\sigma, s} \leq \frac{|h|_{\sigma, s}}{N^{2}} .
$$

Proof. $\mathscr{L}_{1}$ is diagonal in the Fourier basis of $Q: e^{i l_{1} \varphi_{1}} e^{i l_{2} \varphi_{2}}$ with $\left(l_{1}, l_{2}\right) \in \Lambda_{+} \cup \Lambda_{0} \cup \Lambda_{-}$with eigenvalues

$$
d_{l_{1}, l_{2}}= \begin{cases}\left(1+\epsilon\left(-2 a+a^{2} \epsilon\right)\right) l_{2}^{2}, & \text { if } l_{1}+l_{2}=0 \\ a\left(2+a \epsilon^{2}\right) l_{2}^{2}, & \text { if } l_{1}=0\end{cases}
$$

The eigenvalues of $\mathscr{L}_{1}$ restricted to $Q_{2}(N)$ verify $\left|d_{l_{1}, l_{2}}\right| \geq$ $N^{2} / C$, where the constant $C$ depends on $(\epsilon, a)$, and (48) holds.

Fixed points of the nonlinear operator $\mathfrak{P}: Q_{2} \oplus P \rightarrow$ $Q_{2} \oplus P$ defined by

$$
\begin{aligned}
\mathfrak{P}\left(q_{2}, p, q_{1}\right):= & \left(-\mathscr{L}_{1}^{-1} \Pi_{\mathrm{Q}_{2}} f\left(\varphi_{1}, \varphi_{2}, q_{1}+q_{2}+p, \epsilon\right),\right. \\
& \left.-\epsilon \mathscr{L}_{a, \epsilon}^{-1} \Pi_{P} f\left(\varphi_{1}, \varphi_{2}, q_{1}+q_{2}+p, \epsilon\right)\right)
\end{aligned}
$$

are solutions of the $\left(Q_{2}\right)-(P)$-equations. Using the Contraction Mapping Theorem, we can prove the following lemma.

Lemma 7. For any $R>0$, there exist an integer $N_{0}(R) \in \mathbb{N}^{+}$ and positive constants $\epsilon_{0}(R)>0, C_{0}(R)>0$ such that

$$
\begin{gathered}
\forall\left|q_{1}\right|_{H^{1}} \leq 2 R, \quad \forall \epsilon \in \mathscr{B}_{\gamma}, \quad|\epsilon| \gamma^{-1} \leq \epsilon_{0}(R), \\
\forall N \geq N_{0}(R): 0 \leq N \sigma \leq 1,
\end{gathered}
$$

and there exists a unique solution $\left(q_{2}\left(q_{1}\right), p\left(q_{1}\right)\right)$ := $\left(q_{2}\left(\epsilon, N, q_{1}\right), p\left(\epsilon, N, q_{1}\right)\right) \in Q_{2} \oplus P$ of the $\left(Q_{2}\right)-(P)$-equations satisfying

$$
\begin{aligned}
\left|q_{2}\left(\epsilon, N, q_{1}\right)\right|_{\sigma, s} & \leq \frac{C_{0}(R)}{N^{2}}, \\
\left|p\left(\epsilon, N, q_{1}\right)\right|_{\sigma, s} & \leq C_{0}(R)|\epsilon| \gamma^{-1} .
\end{aligned}
$$

Moreover, the map $q_{1} \rightarrow\left(q_{2}\left(q_{1}\right), p\left(q_{1}\right)\right)$ is $C^{1}\left(B_{2 R}, Q_{2} \oplus P\right)$ and

$$
\begin{gathered}
\left|q_{2}^{\prime}\left(q_{1}\right)[h]\right|_{\sigma, s} \leq \frac{C_{0}(R)}{N^{2}}|h|_{H^{1}}, \\
\left|p^{\prime}\left(q_{1}\right)[h]\right|_{\sigma, s} \leq C_{0}(R)|\epsilon| \gamma^{-1}|h|_{H^{1}} \quad \forall h \in Q_{1} .
\end{gathered}
$$

Proof. Let us consider the ball

$$
B:=\left\{\left(q_{2}, p\right) \in Q_{2} \oplus P,\left|q_{2}\right|_{\sigma, s} \leq \rho_{1},|p|_{\sigma, s} \leq \rho_{2}\right\}
$$

with norm $\left|\left(q_{2}, p\right)\right|_{\sigma, s}:=\left|q_{2}\right|_{\sigma, s}+|p|_{\sigma, s}$. We can claim that, under assumptions (51), there exist $\rho_{1}, \rho_{2} \in(0,1)$ such that the map $\left(q_{2}, p\right) \rightarrow \mathfrak{P}\left(q_{2}, p ; q_{1}\right)$ is a contraction mapping in $B$, that is, we have to prove (i) $\left(q_{2}, p\right) \in B \Rightarrow \mathfrak{P}\left(q_{2}, p ; q_{1}\right) \in B$;

(ii) $\left|\mathfrak{P}\left(q_{2}, p ; q_{1}\right)-\mathfrak{P}\left(\widehat{q}_{2}, \widehat{p} ; q_{1}\right)\right|_{\sigma, s} \leq \eta\left|\left(q_{2}, p\right)-\left(\widehat{q}_{2}, \widehat{p}\right)\right|_{\sigma, s}$, $\forall\left(q_{2}, p\right),\left(\hat{q}_{2}, \widehat{p}\right) \in B$,

where the constant $\eta \in(0,1)$. In the following, $\kappa_{i}(i=$ $1,2, \ldots, 5)$ denote different constants. By (48) and the Banach property of $\mathscr{H}_{\sigma, s}$,

$$
\begin{aligned}
\left|\mathfrak{P}_{1}\left(q_{2}, p ; q_{1}\right)\right|_{\sigma, s} & =\left|\mathscr{L}_{1}^{-1} \Pi_{\mathrm{Q}_{2}} f\left(\varphi_{1}, \varphi_{2}, q_{1}+q_{2}+p, \epsilon\right)\right| \\
& \leq \frac{\kappa_{1}}{N^{2}}\left(\left|q_{1}\right|_{\sigma, s}^{3}+\left|q_{2}\right|_{\sigma, s}^{3}+|p|_{\sigma, s}^{3}\right) .
\end{aligned}
$$

Similarly, for $(a, \epsilon) \in \mathscr{B}_{\gamma}$, by (43), we have

$$
\begin{aligned}
\left|\mathfrak{P}_{2}\left(q_{2}, p ; q_{1}\right)\right|_{\sigma, s} & =\left|\epsilon \mathscr{L}_{a, \epsilon}^{-1} \Pi_{P} f\left(\varphi_{1}, \varphi_{2}, q_{1}+q_{2}+p, \epsilon\right)\right| \\
& \leq \frac{\kappa_{2}|\epsilon|}{\gamma}\left(\left|q_{1}\right|_{\sigma, s}^{3}+\left|q_{2}\right|_{\sigma, s}^{3}+|p|_{\sigma, s}^{3}\right) .
\end{aligned}
$$

For all $q_{1} \in Q_{1}(N)$, setting $[x]=\max \{0,|x|\}$, we can get

$$
\begin{aligned}
& \left|q_{1}\right|_{\sigma, s} \\
& =\sum_{\left|l_{2}\right| \leq N, l_{1}=0}\left|\widehat{q}_{0, l_{2}}\right| e^{\left|l_{2}\right| \sigma}+\sum_{\left|l_{2}\right| \leq N, l_{1}+l_{2}=0}\left|\widehat{q}_{-l_{2}, l_{2}}\right| e^{\left|l_{2}\right| \sigma}\left[-l_{2}\right]^{s} \\
& \leq e^{N \sigma}\left(\sum_{\left|l_{2}\right| \leq N, l_{1}=0}\left|\widehat{q}_{l_{1}, l_{2}}\right|\left(\left[-l_{2}-l_{2}^{2}\right]\right)^{s}\right. \\
& \left.+\sum_{\left|l_{2}\right| \leq N, l_{1}+l_{2}=0}\left|\widehat{q}_{-l_{2}, l_{2}}\right|\left[-l_{2}\right]^{s}\right) \\
& \leq \kappa_{3}\left(\left(\sum_{\left|l_{2}\right| \leq N, l_{1}=0}\left|\widehat{q}_{0, l_{2}}\right|^{2}\left[l_{2}\right]^{2}\right)^{1 / 2}\left(\sum_{l_{2} \in \mathbb{Z}} \frac{1}{\left[l_{2}\right]^{2}}\right)^{1 / 2}\right. \\
& +\left(\sum_{\left|l_{2}\right| \leq N, l_{1}+l_{2}=0}\left|\widehat{q}_{-l_{2}, l_{2}}\right|^{2}\left[l_{2}\right]^{2}\right)^{1 / 2} \\
& \left.\times\left(\sum_{l_{2} \in \mathbb{Z}} \frac{1}{\left(\left[l_{2}\right]\right)^{2(1-s)}}\right)^{1 / 2}\right) \\
& \leq \kappa_{4}\left|q_{1}\right|_{H_{1}}
\end{aligned}
$$

whenever $0 \leq N \sigma \leq 1,0 \leq s<1 / 2$. Thus, $\forall\left|q_{1}\right|_{H_{1}} \leq$ $2 R,\left|q_{2}\right|_{\sigma, s} \leq \rho_{1},|p|_{\sigma, s} \leq \rho_{2}$, we get

$$
\begin{aligned}
\left|\mathfrak{P}_{1}\left(q_{2}, p ; q_{1}\right)\right|_{\sigma, s} & \leq \frac{\kappa_{5}}{N^{2}}\left(R^{3}+\rho_{1}^{3}+\rho_{2}^{3}\right), \\
\left|\mathfrak{P}_{2}\left(q_{2}, p ; q_{1}\right)\right|_{\sigma, s} & \leq \frac{\kappa_{5}|\epsilon|}{\gamma}\left(R^{3}+\rho_{1}^{3}+\rho_{2}^{3}\right) .
\end{aligned}
$$

Now, set $C_{0}(R):=\kappa_{5} R^{3}$, and we define $\rho_{1}:=2 C_{0}(R) / N^{3 / 2}$, $\rho_{2}:=2 C_{0}(R)(|\epsilon| / \gamma)$. By the inequality above, there exist 
$N_{0}(R) \in \mathbb{N}^{+}$and $\epsilon_{0}(R)>0$ such that $\forall N \geq N_{0}(R)$ and $\forall|\epsilon| \gamma^{-1} \leq \epsilon_{0}(R)$,

$$
\left|\mathfrak{P}_{1}\left(q_{2}, p ; q_{1}\right)\right|_{\sigma, s} \leq \rho_{1}, \quad\left|\mathfrak{P}_{2}\left(q_{2}, p ; q_{1}\right)\right|_{\sigma, s} \leq \rho_{2} .
$$

So, we get the proof of (i). Item (ii) can be obtained with the same estimates.

By the Contraction Mapping Theorem, there exists a unique fixed point $\left(q_{2}\left(q_{1}\right), p\left(q_{1}\right)\right):=\left(q_{2}\left(\epsilon, N, q_{1}\right)\right.$, $\left.p\left(\epsilon, N, q_{1}\right)\right)$ of $\mathfrak{P}$ in $B$. The bounds of (52) follow by the definition of $\rho_{1}$ and $\rho_{2}$.

Since the map $\mathfrak{P} \in C^{1}\left(Q_{2} \oplus P \times Q_{1} ; Q_{2} \oplus P \times Q_{1}\right)$, the Implicit Function Theorem implies that the map $q_{1} \rightarrow$ $\left(q_{2}\left(\epsilon, N, q_{1}\right), p\left(\epsilon, N, q_{1}\right)\right)$ is $C^{1}$. Differentiating both sides of $\left(q_{2}\left(q_{1}\right), p\left(q_{1}\right)\right)=\mathfrak{P}\left(q_{2}\left(q_{1}\right), p\left(q_{1}\right), q_{1}\right)$, we can get

$$
\begin{aligned}
q_{2}^{\prime}\left(q_{1}\right) & {[h] } \\
= & -\mathscr{L}_{1}^{-1} \Pi_{\mathrm{Q}_{2}}\left(\partial_{v} f\right)\left(\varphi_{1}, \varphi_{2}, q_{1}+q_{2}\left(q_{1}\right)+p\left(q_{1}\right), \epsilon\right) \\
& \times\left(h+q_{2}^{\prime}\left(q_{1}\right)[h]+p^{\prime}\left(q_{1}\right)[h]\right), \\
p^{\prime}\left(q_{1}\right)[h] & \\
= & -\epsilon \mathscr{L}_{\epsilon}^{-1} \Pi_{P}\left(\partial_{v} f\right)\left(\varphi_{1}, \varphi_{2}, q_{1}+q_{2}\left(q_{1}\right)+p\left(q_{1}\right), \epsilon\right) \\
& \times\left(h+q_{2}^{\prime}\left(q_{1}\right)[h]+p^{\prime}\left(q_{1}\right)[h]\right) .
\end{aligned}
$$

Using (43)-(48) and the Banach property of $\mathscr{H}_{\sigma, s}$, we get

$$
\begin{aligned}
& \left|q_{2}^{\prime}\left(q_{1}\right)[h]\right|_{\sigma, s} \\
& \quad \leq \frac{C(R)}{N^{2}}\left(|h|_{\sigma, s}+\left|q_{2}^{\prime}\left(q_{1}\right)[h]\right|_{\sigma, s}+\left|p^{\prime}\left(q_{1}\right)[h]\right|_{\sigma, s}\right), \\
& \left|p^{\prime}\left(q_{1}\right)[h]\right|_{\sigma, s} \\
& \quad \leq \frac{C(R)|\epsilon|}{\gamma}\left(|h|_{\sigma, s}+\left|q_{2}^{\prime}\left(q_{1}\right)[h]\right|_{\sigma, s}+\left|p^{\prime}\left(q_{1}\right)[h]\right|_{\sigma, s}\right),
\end{aligned}
$$

which implies the bounds (53), since when $C(R)\left(|\epsilon| / \gamma+1 / N^{2}\right)$ is small enough, we can get

$$
\operatorname{det}\left|\begin{array}{cc}
1-\frac{C(R)}{N^{2}} & -\frac{C(R)}{N^{2}} \\
-\frac{C(R)|\epsilon|}{\gamma} & 1-\frac{C(R)|\epsilon|}{\gamma}
\end{array}\right| \geq \frac{1}{2} .
$$

3.3. The $\left(Q_{1}\right)$-Equation. Once the $\left(Q_{2}\right)-(P)$-equations have been solved by $\left(q_{2}\left(q_{1}\right), p\left(q_{1}\right)\right) \in Q_{2} \oplus P$, there remains the finite-dimensional $Q_{1}$-equation

$$
\mathscr{L}_{1}\left[q_{1}\right]+\Pi_{\mathrm{Q}_{1}} f\left(\varphi_{1}, \varphi_{2}, q_{1}+q_{2}\left(q_{1}\right)+p\left(q_{1}\right), \epsilon\right)=0 .
$$

The geometric interpretation of the construction of $\left(q_{2}\left(q_{1}\right), p\left(q_{1}\right)\right)$ is that, on the finite-dimensional submanifold
$Z:=\left\{q_{1}+q_{2}\left(q_{1}\right)+p\left(q_{1}\right):\left|q_{1}\right|<2 R\right\}$, diffeomorphic to the ball

$$
B_{2 R}:=\left\{q \in Q_{1}:\left|q_{1}\right|_{H^{1}}<2 R\right\},
$$

the partial derivatives of the action functional $\Psi_{\epsilon}$ with respect to the variables $\left(q_{2}, p\right)$ vanish. We claim that, at a critical point of $\Psi_{\epsilon}$ restricted to $Z$, also the partial derivative of $\Psi_{\epsilon}$ with respect to the variable $q_{1}$ vanishes, and therefore such a point is critical also for the nonrestricted functional $\Psi_{\epsilon}: \mathscr{H}_{\sigma, s} \rightarrow$ $\mathbb{R}$.

Actually, the bifurcation equation (63) is the EulerLagrange equation of the reduced action functional

$$
\begin{gathered}
\Phi_{\epsilon, N}: B_{2 R} \subset Q_{1} \longrightarrow \mathbb{R}, \\
\Phi_{\epsilon, N}\left(q_{1}\right):=\Psi_{\epsilon}\left(q_{1}+q_{2}\left(q_{1}+p\left(q_{1}\right)\right)\right) .
\end{gathered}
$$

Lemma 8. $\Phi_{\epsilon, N} \in C^{1}\left(B_{2 R}, \mathbb{R}\right)$ and a critical point $q_{1} \in B_{2 R}$ of $\Phi_{\epsilon, N}$ is a solution of the bifurcation (63). Moreover, $\Phi_{\epsilon, N}$ can be written as

$$
\Phi_{\epsilon, N}\left(q_{1}\right)=\text { const }+\epsilon\left(\Gamma\left(q_{1}\right)+\Re_{\epsilon, N}\left(q_{1}\right)\right),
$$

where

$$
\begin{array}{rl}
\Gamma\left(q_{1}\right):=\int_{\mathbb{T}^{2}} & \frac{1}{2}\left(\partial_{\varphi_{1}} q_{1}\right)^{2}+\frac{\left(2 a+a^{2} \epsilon^{2}\right)}{2}\left(\partial_{\varphi_{2}} q_{1}\right)^{2} \\
+ & a(1+\epsilon)\left(\partial_{\varphi_{1}} q_{1}\right)\left(\partial_{\varphi_{2}} q_{1}\right)-a_{3}\left(\varphi_{1}, \varphi_{2}\right) \frac{q_{1}^{4}}{4} \\
\mathfrak{R}_{\epsilon, N}\left(q_{1}\right):=\int_{\mathbb{T}^{2}} & F\left(\varphi_{1}, \varphi_{2}, q_{1}, \epsilon=0\right) \\
& -F\left(\varphi_{1}, \varphi_{2}, q_{1}+q_{2}+p, \epsilon\right) \\
+ & \frac{1}{2} f\left(\varphi_{1}, \varphi_{2}, v, \epsilon\right)\left(q_{2}+p\right)
\end{array}
$$

and for some positive constant $C_{2}(R) \geq C_{1}(R)$, we can get

$$
\begin{gathered}
\left|\Re_{\epsilon, N}\left(q_{1}\right)\right| \leq C_{2}(R)\left(\epsilon+\frac{|\epsilon|}{\gamma}+\frac{1}{N^{2}}\right), \\
\left|\Re_{\epsilon, N}^{\prime}\left(q_{1}\right)[h]\right| \leq C_{2}(R)\left(\epsilon+\frac{|\epsilon|}{\gamma}+\frac{1}{N^{2}}\right)|h|_{H^{1}},
\end{gathered}
$$

$\forall h \in Q_{1}$.

Proof. By (37) and (38), we have that, at $v:=q_{1}+q_{2}\left(q_{1}\right)+$ $p\left(q_{1}\right)$,

$$
\begin{array}{ll}
d \Psi_{\epsilon}(v)[h]=0, & \forall h \in Q_{2}, \\
d \Psi_{\epsilon}(v)[h]=0, & \forall h \in P .
\end{array}
$$

Since $q_{2}^{\prime}\left(q_{1}\right)[h] \in Q_{2}$ and $p^{\prime}\left(q_{1}\right)[h] \in P, \forall h \in Q_{1}$, we deduce that

$$
\begin{aligned}
d \Phi_{\epsilon, N}\left(q_{1}\right)[h] & =d \Psi_{\epsilon}(v)\left[[h]+q_{2}^{\prime}\left(q_{1}\right)[h]+p^{\prime}\left(q_{1}\right)[h]\right] \\
& =d \Psi_{\epsilon}(v)[h], \quad \forall h \in Q_{1},
\end{aligned}
$$


and therefore $v:=q_{1}+q_{2}\left(q_{1}\right)+p\left(q_{1}\right)$ solves also the $\left(Q_{1}\right)$ equation (36). Write $\Psi_{\epsilon}(v):=\Psi_{\epsilon}^{(2)}(v)-\epsilon \int_{\mathbb{T}^{2}} F\left(\varphi_{1}, v, \epsilon\right)$, where

$$
\begin{aligned}
& \Psi_{\epsilon}^{(2)}(v)=\int_{\mathbb{T}^{2}}\left(1+\frac{1}{2} \epsilon\right)\left(\partial_{\varphi_{1}} v\right)^{2}+\epsilon \frac{2 a+a^{2} \epsilon^{2}}{2}\left(\partial_{\varphi_{2}} v\right)^{2} \\
&+(1+\epsilon a(1+\epsilon))\left(\partial_{\varphi_{1}} v\right)\left(\partial_{\varphi_{2}} v\right)
\end{aligned}
$$

is a homogeneous functional of degree two. By homogeneity,

$$
\Psi_{\epsilon}(v)=\frac{1}{2} d \Psi_{\epsilon}^{(2)}(v)[v]-\epsilon \int_{\mathbb{T}^{2}} F\left(\varphi_{1}, \varphi_{2}, v, \epsilon\right)
$$

and, according to (69), we have

$$
\begin{gathered}
d \Psi_{\epsilon}^{(2)}\left(q_{1}+q_{2}\left(q_{1}\right)+p\left(q_{1}\right)\right)\left[q_{2}\left(q_{1}\right)+p\left(q_{1}\right)\right] \\
=\epsilon \int_{\mathbb{T}^{2}} f\left(\varphi_{1}, \varphi_{2}, v, \epsilon\right)\left(q_{2}\left(q_{1}\right)+p\left(q_{1}\right)\right) .
\end{gathered}
$$

Substituting the above equality into (72), we obtain, at $v:=$ $q_{1}+q_{2}\left(q_{1}\right)+p\left(q_{1}\right)$

$$
\begin{aligned}
\Phi_{\epsilon, N}\left(q_{1}\right)= & \Psi_{\epsilon}\left(q_{1}+q_{2}\left(q_{1}\right)+p\left(q_{1}\right)\right) \\
= & \frac{1}{2} d \Psi_{\epsilon}^{(2)}(v)\left[q_{1}+q_{2}\left(q_{1}\right)+p\left(q_{1}\right)\right] \\
& -\epsilon \int_{\mathbb{T}^{2}} F\left(\varphi_{1}, \varphi_{2}, v, \epsilon\right) \\
= & \frac{1}{2} d \Psi_{\epsilon}^{(2)}\left(q_{1}\right)\left[q_{1}\right] \\
& +\frac{1}{2} \epsilon \int_{\mathbb{T}^{2}} f\left(\varphi_{1}, \varphi_{2}, v, \epsilon\right)\left(q_{2}\left(q_{1}\right)+p\left(q_{1}\right)\right) \\
& -\epsilon \int_{\mathbb{T}^{2}} F\left(\varphi_{1}, \varphi_{2}, v, \epsilon\right) \\
= & \int_{\mathbb{T}^{2}}\left(\partial_{\varphi_{1}} q_{1}\right)^{2}+\left(\partial_{\varphi_{1}} q_{1}\right)\left(\partial_{\varphi_{2}} q_{1}\right) \\
& +\epsilon\left(\int_{\mathbb{T}^{2}} \frac{1}{2}\left(\partial_{\varphi_{1}} q_{1}\right)^{2}+\frac{\left(2 a+a^{2} \epsilon^{2}\right)}{2}\left(\partial_{\varphi_{2}} q_{1}\right)^{2}\right. \\
+ & \Psi_{0}\left(q_{1}\right) \\
& +F(1+\epsilon)\left(\partial_{\varphi_{1}} q_{1}\right)\left(\partial_{\varphi_{2}} q_{1}\right) \\
& \left.+\frac{1}{2} f\left(\varphi_{1}, \varphi_{2}, v, \epsilon\right)\left(q_{2}\left(q_{1}\right)+p\left(q_{1}\right)^{2}, v, \epsilon\right)\right) \\
& \\
& \left.+\partial_{\varphi_{2}} q_{1}\right)^{2} \\
& \\
& \\
& \\
& \\
&
\end{aligned}
$$

$$
\begin{aligned}
& +a(1+\epsilon)\left(\partial_{\varphi_{1}} q_{1}\right)\left(\partial_{\varphi_{2}} q_{1}\right) \\
& +\frac{1}{2} f\left(\varphi_{1}, \varphi_{2}, v, \epsilon\right)\left(q_{2}\left(q_{1}\right)+p\left(q_{1}\right)\right) \\
& \left.-F\left(\varphi_{1}, \varphi_{2}, v, \epsilon\right)\right) .
\end{aligned}
$$

Because $\Psi_{0}\left(q_{1}\right) \equiv$ const, by (52), we can get the bounds of (68).

The problem of finding nontrivial solutions of the $Q_{1}$ equation is reduced to finding nontrivial critical points of the reduced action functional $\Phi_{\epsilon, N}$ in $B_{2 R}$. By (66), this is equivalent to finding critical points of the rescaled functional

$$
\begin{aligned}
\widehat{\Phi}_{\epsilon, N} & =\Gamma\left(q_{1}\right)+\mathfrak{R}_{\epsilon, N}\left(q_{1}\right) \\
& =\left(\mathfrak{Q}\left(q_{1}\right)-\int_{\mathbb{T}^{2}} a_{3}\left(\varphi_{1}, \varphi_{2}\right) \frac{q_{1}^{4}}{4}\right)+\mathfrak{R}_{\epsilon, N}\left(q_{1}\right),
\end{aligned}
$$

where the quadratic form

$$
\begin{aligned}
\mathfrak{Q}(q):=\int_{\mathbb{T}^{2}} & \frac{1}{2}\left(\partial_{\varphi_{1}} q_{1}\right)^{2}+\frac{\left(2 a+a^{2} \epsilon^{2}\right)}{2}\left(\partial_{\varphi_{2}} q_{1}\right)^{2} \\
& +a(1+\epsilon)\left(\partial_{\varphi_{1}} q_{1}\right)\left(\partial_{\varphi_{2}} q_{1}\right)
\end{aligned}
$$

is positive definite on $Q_{+}$, negative definite on $Q_{-}$, and zero definite on $Q_{0}$. For $q_{1}=q_{+}+q_{-}+q_{0} \in Q_{1}$ and $a<0$, we have

$$
\begin{aligned}
\mathfrak{Q}\left(q_{+}\right)= & \int_{\mathbb{T}^{2}} \frac{1}{2}\left(\partial_{\varphi_{1}} q_{+}\right)^{2}+\frac{\left(2 a+a^{2} \epsilon^{2}\right)}{2}\left(\partial_{\varphi_{2}} q_{+}\right)^{2} \\
& +a(1+\epsilon)\left(\partial_{\varphi_{1}} q_{+}\right)\left(\partial_{\varphi_{2}} q_{+}\right) \\
= & \int_{\mathbb{T}^{2}} \sum_{\left(l_{1}, l_{2}\right) \in \mathbb{Z}^{2}} l_{2}^{2}\left(-a-\frac{a^{2} \epsilon^{2}}{2}\right) \widehat{q}_{l_{1}, l_{2}} e^{i l_{1} \varphi_{1}} e^{i l_{2} \varphi_{2}} \\
= & \frac{\alpha_{+}(a)}{2}\left|q_{+}\right|_{H^{1}}^{2}, \\
\mathfrak{Q}\left(q_{0}\right)= & 0, \quad \sum_{\mathbb{T}^{2}} \sum_{\left(l_{1}, l_{2}\right) \epsilon \mathbb{Z}^{2}} \frac{(1+a \epsilon)^{2}}{2} \widehat{q}_{l_{1}, l_{2}} e^{i l_{1} \varphi_{1}} e^{i l_{2} \varphi_{2}} \\
\mathfrak{Q}\left(q_{-}\right)= & -\frac{\alpha_{-}(a)}{2}\left|q_{-}\right|_{H^{1}}^{2},
\end{aligned}
$$

where the positive constants $\alpha_{+}(a), \alpha_{-}(a)$ are bounded away from zero and independent of $\epsilon$. We will prove the existence of critical point of $\widehat{\Phi}_{\epsilon, N}$ in $B_{2 R}$ of linking type.

\subsection{Linking Critical Points of the Reduced Action Functional $\widehat{\Phi}_{\epsilon, N}$. We cannot directly apply the linking theorem because $\widehat{\Phi}_{\epsilon, N}$ is defined only in the ball $B_{2 R}$. Therefore, we first extend}


$\widehat{\Phi}_{\epsilon, N}$ to the whole space $Q_{1}$. We define the extended action function $\widetilde{\Phi}_{\epsilon, N} \in C^{1}\left(Q_{1}, \mathbb{R}\right)$ as

$$
\widetilde{\Phi}_{\epsilon, N}\left(q_{1}\right)=\Gamma\left(q_{1}\right)+\widetilde{\Re}_{\epsilon, N}\left(q_{1}\right),
$$

where $\widetilde{\mathfrak{R}}_{\epsilon, N}\left(q_{1}\right): Q_{1} \rightarrow \mathbb{R}$ is

$$
\widetilde{\mathfrak{R}}_{\epsilon, N}\left(q_{1}\right):=\lambda\left(\frac{\left|q_{1}\right|_{H^{1}}^{2}}{R^{2}}\right) \mathfrak{R}_{\epsilon, N}\left(q_{1}\right),
$$

and $\lambda:[0,+\infty) \rightarrow[0,1]$ is a smooth, nonincreasing, cut-off function such that

$$
\begin{gathered}
\lambda(x)=1, \quad \text { if }|x| \leq 1 ; \\
\lambda(x)=0, \quad \text { if }|x| \geq 4, \quad\left|\lambda^{\prime}(x)\right|<1 .
\end{gathered}
$$

By definition, $\widetilde{\Phi}_{\epsilon, N}\left(q_{1}\right) \equiv \widehat{\Phi}_{\epsilon, N}\left(q_{1}\right)$ on $B_{R}:=\left\{q_{1} \in Q_{1}\right.$ : $\left.\left|q_{1}\right|_{H^{1}} \leq R\right\}$ and $\widetilde{\Phi}_{\epsilon, N}\left(q_{1}\right)=\Gamma\left(q_{1}\right)$ outside $B_{2 R}$. Moreover, by (68), there is a constant $C_{3}(R) \geq C_{2}(R)>0$ such that $\forall|q|_{H^{1}} \leq 2 R$, and

$$
\begin{array}{r}
\left|\widetilde{\Re}_{\epsilon, N}\left(q_{1}\right)\right| \leq C_{3}(R)\left(\epsilon+|\epsilon| \gamma^{-1}+\frac{1}{N^{2}}\right), \\
\left|\widetilde{R}_{\epsilon, N}^{\prime}\left(q_{1}\right)[h]\right| \leq C_{3}(R)\left(\epsilon+|\epsilon| \gamma^{-1}+\frac{1}{N^{2}}\right)|h|_{H^{1}}, \\
\forall h \in Q_{1} .
\end{array}
$$

Second, we will verify that $\widetilde{\Phi}_{\epsilon, N}\left(q_{1}\right)$ satisfies the geometrical hypotheses of the linking theorem.

Lemma 9. There exist positive constants $\rho, \beta, r_{1}, r_{2}>\rho$, and $0<\epsilon_{1}(R) \leq \epsilon_{0}(R), N_{1}(R) \geq N_{0}(R)$, which are independent of $(\epsilon, N, \gamma)$, such that, $\forall(|\epsilon| / \gamma) \leq \epsilon_{1}$ and $\forall N \geq N_{1}(R)$,

(i) $\inf _{q_{1} \in S^{+}} \widetilde{\Phi}_{\epsilon, N}\left(q_{1}\right) \geq \beta>0, \forall q_{1} \in S^{+}:=\left\{q_{1} \in Q_{1} \cap Q_{+}\right.$: $\left.\left|q_{1}\right|_{H^{1}}=\rho\right\}$,

(ii) $\sum_{q_{1} \in \partial W^{-}} \widetilde{\Phi}_{\epsilon, N}\left(q_{1}\right) \leq \beta / 2, \forall q_{1} \in \partial W^{-}$,

where $W^{-}$is the rectangle in $Q_{-} \oplus Q_{0}$,

$$
\begin{aligned}
W^{-}:=\left\{q_{1}=\right. & q_{0}+q_{+}+r e^{+},\left|q_{0}+q_{-}\right| \leq r_{1}, q_{-} \in Q_{1} \cap Q_{-}, \\
& \left.q_{0} \in \mathbb{R}, r \in\left[0, r_{2}\right]\right\},
\end{aligned}
$$

and $e_{+}$is the unit vector in $Q_{1} \oplus Q_{0}$.

Proof. (i) $\forall q_{+} \in Q_{1} \cap Q_{+}$with $\left|q_{+}\right|_{H^{1}}=\rho<R$, we have

$$
\begin{aligned}
\widetilde{\Phi}_{\epsilon, N}\left(q_{+}\right) & =\widehat{\Phi}_{\epsilon, N}\left(q_{+}\right) \\
& =\mathfrak{Q}\left(q_{+}\right)-\int_{\mathbb{T}^{2}} a_{3}\left(\varphi_{1}, \varphi_{2}\right) \frac{q_{+}^{4}}{4}+\Re_{\epsilon, N}\left(q_{+}\right) \\
& \geq \frac{\alpha_{+}(a)}{2} \rho^{2}-\kappa_{1} \rho^{4}-C_{3}(R)\left(\epsilon+\frac{\epsilon}{\gamma}+\frac{1}{N^{2}}\right) .
\end{aligned}
$$

Now, we fix $\rho>0$ small such that $\left(\alpha_{+}(a) / 2\right) \rho^{2}-\kappa_{1} \rho^{4} \geq$ $\left(\alpha_{+}(a) / 4\right) \rho^{2}$. Since $C_{3}(R)\left(\epsilon+\epsilon / \gamma+1 / N^{2}\right) \leq\left(\alpha_{+}(a) / 8\right) \rho^{2}$, by (84), we can get

$$
\begin{array}{r}
\widetilde{\Phi}_{\epsilon, N}\left(q_{+}\right) \geq \frac{\alpha_{+}(a)}{8} \rho^{2}:=\beta>0, \quad \forall q_{+} \in Q_{1} \cap Q_{+}, \\
\text {with }\left|q_{+}\right|_{H^{1}}=\rho .
\end{array}
$$

(ii) Let

$$
\begin{aligned}
B_{1}:= & \left\{q_{1}=q_{0}+q_{-}+r_{2} e_{+} \text {with }\left|q_{0}+q_{-}\right|_{H^{1}} \leq r_{1},\right. \\
& \left.q-\in Q_{1} \cap Q_{-}\right\} \subset \partial W^{-}, \\
B_{2}:= & \left\{q_{1}=q_{0}+q_{-}+r e_{+} \text {with }\left|q_{0}+q_{-}\right|_{H^{1}}=r_{1},\right. \\
& \left.q-\in Q_{1} \cap Q_{-}, r \in\left[0, r_{2}\right]\right\} \subset \partial W^{-},
\end{aligned}
$$

with $r_{1}, r_{2}>2 R$. For $q_{1}=q_{0}+q_{-}+r e_{+} \in B_{1} \cup B_{2}$,

$$
\begin{aligned}
\widetilde{\Phi}_{\epsilon, N}\left(q_{1}\right)= & \gamma\left(q_{1}\right) \\
= & \mathfrak{Q}\left(q_{1}\right)-\int_{\mathbb{T}^{2}} a_{3}\left(\varphi_{1}, \varphi_{2}\right) \frac{\left(q_{0}+q_{-}+r e_{+}\right)^{4}}{4} \\
\leq & -\frac{\alpha_{-}(a)}{2}\left|q_{-}\right|_{H^{1}}^{2}+r^{2} \mathfrak{Q}\left(e_{+}\right) \\
& -\int_{\mathbb{T}^{2}} a_{3}\left(\varphi_{1}, \varphi_{2}\right) \frac{\left(q_{0}+q_{-}+r e_{+}\right)^{4}}{4} \\
\leq & -\frac{\alpha_{-}(a)}{2}\left|q_{-}\right|_{H^{1}}^{2}+r^{2} \mathfrak{Q}\left(e_{+}\right) \\
& -\alpha \int_{\mathbb{T}^{2}}\left(q_{0}+q_{-}+r e_{+}\right)^{4},
\end{aligned}
$$

because $a_{3}\left(\varphi_{1}, \varphi_{2}\right) / 4 \geq \alpha>0$. Now, by Hölder inequality and orthogonality,

$$
\begin{aligned}
\int_{\mathbb{T}^{2}}\left(q_{0}+q_{-}+r e_{+}\right)^{4} & \geq \kappa_{2}\left(\int_{\mathbb{T}^{2}}\left(q_{0}+q_{-}+r e_{+}\right)^{2}\right)^{2} \\
& =\kappa_{2}\left(q_{0}^{2}+q_{-}^{2}+r^{2} e_{+}^{2}\right)^{2} \\
& \geq \kappa_{3}\left(q_{0}^{2}+r^{2}\right)^{2} \geq \kappa_{3}\left(q_{0}^{4}+r^{4}\right),
\end{aligned}
$$

and by (88) we deduce that

$$
\begin{aligned}
\widetilde{\Phi}_{\epsilon, N}\left(q_{0}+q_{-}+r e_{+}\right) \leq & \left(\kappa_{4} r^{2}-\kappa_{3} r^{4}\right) \\
& -\left(\frac{\alpha_{-}(a)}{2}\left|q_{-}\right|_{H^{1}}^{2}+\kappa_{3} q_{0}^{4}\right) .
\end{aligned}
$$

Now, we fix $r_{2}$ large such that $\kappa_{4} r_{2}^{2}-\kappa_{3} r_{2}^{4} \leq 0$, and therefore

$$
\widetilde{\Phi}_{\epsilon, N}\left(q_{1}\right) \leq \kappa_{4} r_{2}^{2}-\kappa_{3} r_{2}^{4} \leq 0, \quad \forall q_{1} \in B_{1} .
$$

Next, setting $M:=\max _{\left[0, r_{2}\right]} \kappa_{4} r^{2}-\kappa_{3} r^{4}$, we fix $r_{1}$ large such that

$$
\frac{\alpha_{-}(a)}{2}\left|q_{-}\right|_{H^{1}}^{2}+\kappa_{3} q_{0}^{4} \geq M, \quad \forall\left|q_{-}+q_{0}\right|=r_{1},
$$


and therefore

$$
\widetilde{\Phi}_{\epsilon, N}\left(q_{1}\right) \leq M-\left(\frac{\alpha_{-}(a)}{2}\left|q_{-}\right|_{H^{1}}^{2}+\kappa_{3} q_{0}^{4}\right) \leq 0, \quad \forall q_{1} \in B_{2} .
$$

Finally, if $q_{1}=q_{-}+q_{0}$,

$$
\begin{aligned}
\widetilde{\Phi}_{\epsilon, N}\left(q_{1}\right) & =\mathfrak{Q}\left(q_{-}\right)-\int_{\mathbb{T}^{2}} a_{3}\left(\varphi_{1}, \varphi_{2}\right) \frac{q_{1}^{4}}{4}+\widetilde{\mathfrak{R}}_{\epsilon, N}\left(q_{1}\right) \\
& \leq\left|\widetilde{\Re}_{\epsilon, N}\left(q_{1}\right)\right| \leq C_{3}(R)\left(\epsilon+|\epsilon| \gamma^{-1}+\frac{1}{N^{2}}\right) .
\end{aligned}
$$

So, if $C_{3}(R)\left(\sqrt{\epsilon}+|\epsilon| \gamma^{-1}+\left(1 / N^{2}\right)\right) \leq \beta / 2$, we can get $\widetilde{\Phi}_{\epsilon, N}\left(q_{1}\right) \leq$ $\beta / 2, \forall q_{1} \in \partial W^{-}$.

We introduce the minimax class $\mathcal{S}:=\left\{\psi \in C\left(\bar{W}^{-}, Q\right) \mid\right.$ $\psi=$ Id on $\left.\partial W_{-}\right\}$. According to Proposition 5.9 of [19], the maps of $\mathcal{S}$ have an important intersection property as follows.

Proposition 10. $\left(S^{+}\right.$and $W^{-}$link with respect to $\left.\psi\right)$. Consider

$$
\psi \in \mathcal{S} \Longrightarrow \psi\left(W^{-}\right) \cap S^{+} \neq \emptyset \text {. }
$$

One defines the minimax linking level as follows:

$$
\widetilde{c}_{\epsilon}:=\inf _{\psi \in \mathcal{S} q_{q_{1}} \in W^{-}} \widetilde{\Phi}_{\epsilon, N}\left(\psi\left(q_{1}\right)\right) .
$$

Obviously, by Proposition 10 and Lemma 9,

$$
\max _{q_{1} \in W^{-}} \widetilde{\Phi}_{\epsilon, N}\left(\psi\left(q_{1}\right)\right) \geq \min _{q_{1} \in S^{+}} \widetilde{\Phi}_{\epsilon, N}\left(q_{1}\right) \geq \beta>0, \quad \forall \psi \in \mathcal{S},
$$

and, therefore, $\widetilde{c}_{\epsilon}>\beta>0$.

Since Id $\in \mathcal{S}$, so

$$
\begin{aligned}
\widetilde{\mathcal{c}}_{\epsilon} \leq \max _{q \in W^{-}} \widetilde{\Phi}_{\epsilon, N}\left(\left(q_{1}\right)\right) \leq \max _{q \in W^{-}}\left(\Gamma\left(q_{1}\right)+\widetilde{\Re}_{\epsilon, N}\left(q_{1}\right)\right) \\
\leq \max _{q \in W^{-}}\left(\frac{\alpha_{+}(a)}{2}\left|q_{+}\right|_{H^{1}}^{2}+\frac{\alpha_{-}(a)}{2}\left|q_{-}\right|_{H^{1}}^{2}+\int_{\mathbb{T}^{2}} \kappa q_{1}^{4}\right) \\
\quad+1 \leq \widetilde{c}_{\infty} \leq+\infty,
\end{aligned}
$$

where $\widetilde{c}_{\infty}$ is independent of $N, \epsilon, \gamma$. By the linking theorem, we deduce the existence of a Palais-Smale sequence $\left(q_{n}\right) \in Q_{1}$ at the level $\widetilde{c}_{\epsilon}$, namely,

$$
\widetilde{\Phi}_{\epsilon, N}\left(q_{n}\right) \longrightarrow \widetilde{c}_{\epsilon}, \quad \widetilde{\Phi}_{\epsilon, N}^{\prime}\left(q_{n}\right) \longrightarrow 0 \quad \text { when } n \longrightarrow \infty .
$$

Third, we will prove that the Palais-Smale sequence $\left(q_{n}\right)$ (up to subsequence) converges to some nontrivial critical point $\widehat{q}_{1} \neq 0$ in some open ball of $Q_{1}$, where $\widetilde{\Phi}_{\epsilon, N}$ and $\widehat{\Phi}_{\epsilon, N}$ coincide. Because the space $Q_{1}$ is finite dimensional, we only need to prove that the sequence $\left(q_{n}\right)$ is bounded.

Lemma 11. There is a constant $M>0$ independent of $R, \epsilon, N, \gamma$, such that, for all $|\epsilon| / \gamma$ small enough and $N$ large enough, the Palais-Smale sequence $\left(q_{n}\right)$ is bounded; that is, $\left|q_{n}\right|_{H^{1}}<M$. So, there exists a subsequence of the P-S sequence that converges to some critical point $\widehat{q}_{1} \neq 0$, and the functional $\widetilde{\Phi}_{\epsilon, N}$ possesses a nontrivial critical point $\widehat{q}_{1} \in Q_{1}$ with critical value $\widetilde{\Phi}_{\epsilon, N}\left(\widehat{q}_{1}\right)=\widetilde{c}_{\epsilon}$.
Proof. In the sequel, we will always assume that $\left(\epsilon+|\epsilon| \gamma^{-1}+\right.$ $\left.1 / N^{2}\right)<1$. Writing $\widetilde{\Phi}_{\epsilon, N}(q)=\Gamma(q)+\widetilde{\Re}_{\epsilon, N}(q)$, by $(81)-(82)$, we can get

$$
\begin{aligned}
\widetilde{\Phi}_{\epsilon, N} & \left(q_{n}\right)-\frac{1}{2} \widetilde{\Phi}_{\epsilon, N}^{\prime}\left(q_{n}\right)\left[q_{n}\right] \\
= & \Gamma\left(q_{n}\right)-\frac{1}{2} \Gamma^{\prime}\left(q_{n}\right)\left[q_{n}\right] \\
& +\left(\widetilde{\Re}_{\epsilon, N}\left(q_{n}\right)-\frac{1}{2} \widetilde{\Re}_{\epsilon, N}^{\prime}\left(q_{n}\right)\left[q_{n}\right]\right) \\
= & \left(\frac{1}{2}-\frac{1}{4}\right) \int_{\mathbb{T}^{2}} a_{3}\left(\varphi_{1}\right) q_{j}^{4} \\
& +\left(\widetilde{\Re}_{\epsilon, N}\left(q_{n}\right)-\frac{1}{2} \widetilde{\Re}_{\epsilon, N}^{\prime}\left(q_{n}\right)\left[q_{n}\right]\right) \\
\geq & \alpha\left(\frac{1}{2}-\frac{1}{4}\right) \int_{\mathbb{T}^{2}} q_{j}^{4}-\left(\epsilon+|\epsilon| \gamma^{-1}+\frac{1}{N^{2}}\right) .
\end{aligned}
$$

Then, by $\widetilde{c}_{\epsilon}<\widetilde{c}_{\infty}<+\infty$ and $\widetilde{\Phi}_{\epsilon, N}^{\prime}\left(q_{n}\right) \rightarrow 0(n \rightarrow \infty)$,

$$
\widetilde{c}_{\infty}+1+\left|q_{n}\right|_{H^{1}} \geq \kappa_{6} \int_{\mathbb{T}^{2}} q_{n}^{4}:=\kappa_{6}\left|q_{n}\right|_{L^{4}}^{4}
$$

By Hölder inequality and orthogonality,

$$
\begin{aligned}
\tilde{c}_{\infty}+1+\left|q_{n}\right|_{H^{1}} & \geq \kappa_{7}\left(\int_{\mathbb{T}^{2}}\left(q_{0, n}+q_{-, n}+q_{+, n}\right)^{2}\right)^{2} \\
& =\kappa_{7}\left(\int_{\mathbb{T}^{2}} q_{0, n}^{2}+q_{-, n}^{2}+q_{+, n}^{2}\right)^{2} \geq \kappa_{8}\left(q_{0, n}\right)^{4},
\end{aligned}
$$

and therefore $\left|q_{0, n}\right| \leq\left(1+\left|q_{n}\right|_{H^{1}}\right)^{1 / 4}$. In the same way, by Hölder inequality and (82),

$$
\begin{aligned}
\widetilde{\Phi}_{\epsilon, N}^{\prime}\left(q_{n}\right)\left[q_{+, n}\right]= & \alpha_{+}(a)\left|q_{+, n}\right|_{H_{1}}^{2} \\
& -\int_{\mathbb{T}^{2}} a_{3}\left(\varphi_{1}\right) q_{n}^{3} q_{+, n}+\widetilde{\Re}_{\epsilon, N}^{\prime}\left(q_{n}\right)\left[q_{+, n}\right] \\
\geq & \alpha_{+}(a)\left|q_{+, n}\right|_{H_{1}}^{2}-\kappa_{9}\left|q_{+, n}\right|_{H_{1}} \int_{\mathbb{T}^{2}}\left|q_{n}\right|^{3} \\
& -C_{3}(R)\left(\sqrt{\epsilon}+|\epsilon| \gamma^{-1}+\frac{1}{N^{3 / 2}}\right)\left|q_{+, n}\right|_{H_{1}} \\
\geq & \kappa_{10}\left|q_{+, n}\right|_{H_{1}}\left(\left|q_{+, n}\right|_{H_{1}}-\left|q_{n}\right|_{L^{4}}^{3}-1\right) .
\end{aligned}
$$

By (101) and the above inequalities, using $\widetilde{\Phi}_{\epsilon, N}^{\prime}\left(q_{n}\right) \rightarrow$ $0(n \rightarrow \infty)$, we conclude that $\left|q_{+, n}\right|_{H_{1}} \leq \kappa_{11}\left(1+\left|q_{n}\right|_{H_{1}}^{3 / 4}\right)$. Estimating analogously, we derive $\left|q_{-, n}\right|_{H_{1}} \leq \kappa_{12}\left(1+\left|q_{n}\right|_{H_{1}}^{3 / 4}\right)$. Finally, we deduce that

$$
\begin{aligned}
\left|q_{n}\right|_{H_{1}} & =\left|q_{0, n}\right|+\left|q_{+, n}\right|_{H_{1}}+\left|q_{-, n}\right|_{H_{1}} \\
& \leq \kappa_{13}\left(1+\left|q_{n}\right|_{H_{1}}^{3 / 4}+\left|q_{n}\right|_{H_{1}}^{3 / 4}\right) .
\end{aligned}
$$


So, we can conclude that $\left|q_{n}\right|_{H_{1}} \leq M$ for a suitable constant $M>0$, which is independent of $R, \epsilon, N, \gamma$. Since $Q_{1}$ is finite dimensional, $\left\{q_{n}\right\}$ converges, up to subsequence, to some critical point $\widehat{q}$ of $\widetilde{\Phi}_{\epsilon, N}$ with $|\widehat{q}|_{H_{1}}<M$. Since $\widetilde{\Phi}_{\epsilon, N}(\widehat{q})=\widetilde{c}_{\epsilon} \geq$ $\beta>0$, we conclude that $\hat{q} \neq 0$.

Proof of Theorem 1. Let us fix $\bar{R}:=M+1$ and take $|\epsilon| \gamma^{-1} \leq$ $\epsilon_{2}(\bar{R}):=\bar{\epsilon}$. According to Lemma 7, we can get, for $0<$ $\sigma N(\bar{R}) \leq 1,0 \leq s<1 / 2$, a solution $\left(q_{2}\left(q_{1}\right), p\left(q_{1}\right)\right) \in$ $\left(Q_{2}(\bar{N}) \oplus P\right) \cap \mathscr{H}_{\sigma, s}$ of the $\left(Q_{2}\right)-(P)$-equations with $\forall\left|q_{1}\right|_{H_{1}} \leq$ $2 R$. By Lemma 11, the extended functional $\widetilde{\Phi}_{\epsilon, N}\left(q_{1}\right)$ possesses a nontrivial critical point $\hat{q}$ with $\left|q_{1}\right|_{H_{1}} \leq M<\bar{R}$. Since $\widetilde{\Phi}_{\epsilon, N}$ coincides with $\Phi_{\epsilon, N}$ on the ball $B_{\bar{R}}$ by Lemma 8 , there exists a nontrivial weak solution $\widehat{q}_{1}+q_{2}\left(\widehat{q}_{1}\right)+p\left(\widehat{q}_{1}\right) \in \mathscr{H}_{\sigma, s}$ of (23). Finally, $u=\epsilon\left[\widehat{q}_{1}+q_{2}\left(\widehat{q}_{1}\right)+p\left(\widehat{q}_{1}\right)\right]=\epsilon\left[\widehat{q}_{\epsilon}+p\left(\widehat{q}_{1}\right)\right]$ solves $(7)$.

According to Lemma 6 , by the regularizing property of the operator $\mathscr{L}_{1}$, the solution $\widehat{q}_{\epsilon}:=\widehat{q}_{1}+q_{2}\left(\widehat{q}_{1}\right)$ of the $(Q)$ equation belongs to $\mathscr{H}_{\sigma, s+2} \cap Q$. By the $P$-equation

$$
\begin{aligned}
& \left((2+\epsilon) \partial_{\varphi_{1}}^{2}+2\left(1+a\left(\epsilon+\epsilon^{2}\right)\right) \partial_{\varphi_{1}} \partial_{\varphi_{2}}\right) \widehat{p} \\
& \quad=-\epsilon\left(\left(2 a+a^{2} \epsilon^{2}\right) \partial_{\varphi_{2}}^{2}+\Pi_{P} f\left(\varphi_{1}, \varphi_{2}, \widehat{q}_{\epsilon}+p, \epsilon\right)\right) \widehat{p}
\end{aligned}
$$

where $\widehat{p}=p\left(\widehat{q}_{1}\right)$, we can get that

$$
-\epsilon\left(\left(2 a+a^{2} \epsilon^{2}\right) \partial_{\varphi_{2}}^{2}+\Pi_{P} f\left(\varphi_{1}, \varphi_{2}, \widehat{q}_{\epsilon}+p, \epsilon\right)\right) \hat{p} \in \mathscr{H}_{\sigma \prime, s},
$$

for $0<\sigma^{\prime}<\sigma$, satisfying $\left|\epsilon\left(2 a+a^{2} \epsilon^{2}\right) l_{2}^{2}\right| e^{\left|l_{2}\right| \sigma^{\prime}}<e^{\left|l_{2}\right| \sigma}$. For $(a, \epsilon) \in \mathscr{B}_{\gamma}$, the eigenvalues of operator $(2+\epsilon) \partial_{\varphi_{1}}^{2}+2(1+a(\epsilon+$ $\left.\left.\epsilon^{2}\right)\right) \partial_{\varphi_{1}} \partial_{\varphi_{2}}$ restricted to $P$ satisfy $\left|(2+\epsilon) l_{1}^{2}+2\left(1+a \epsilon+a \epsilon^{2}\right) l_{1} l_{2}\right| \geq$ $|2+\epsilon|\left(\gamma\left|l_{1}\right| /\left|l_{2}\right|\right), \forall l_{1} \neq 0, l_{1}+l_{2} \neq 0$, and, thus, we deduce that $\widehat{p} \in \mathscr{H}_{\sigma^{\prime \prime}, s+1}$, for all $0<\sigma^{\prime \prime}<\sigma^{\prime}$ (satisfying $C\left|l_{2}\right| e^{\left|l_{2}\right| \sigma^{\prime \prime}}<$ $\left.e^{\left|l_{2}\right| \sigma^{\prime}}\right)$, and $\left|\partial_{\varphi_{1}} \widehat{p}\right|_{\sigma^{\prime \prime}, S}=O(|\epsilon| / \gamma)$. By (105),

$$
\begin{aligned}
(2+\epsilon) & \partial_{\varphi_{1}}^{2} \widehat{p} \\
= & -2\left(1+a\left(\epsilon+\epsilon^{2}\right)\right) \partial_{\varphi_{1}} \partial_{\varphi_{2}} \widehat{p} \\
& \quad-\epsilon\left(\left(2 a+a^{2} \epsilon^{2}\right) \partial_{\varphi_{2}}^{2}+\Pi_{P} f\left(\varphi_{1}, \varphi_{2}, \widehat{q}_{\epsilon}+p, \epsilon\right)\right) \hat{p}
\end{aligned}
$$

we get $\widehat{p} \in \mathscr{H}_{\bar{\sigma}, s+2}$, and $|\widehat{p}|_{\bar{\sigma}, s+2}=O\left(|\epsilon| \gamma^{-1}\right)$, with $0<\bar{\sigma}<\sigma^{\prime \prime}$. Thus, (15) follows with $\bar{s}=s+2,0<\bar{\sigma}<\sigma^{\prime \prime}$. By $(5), u(t, x)=$ $\epsilon v\left((1+\epsilon) t+x,\left(1+a \epsilon^{2}\right) t+x\right)$ is the solution of $(1)$, for all $(a, \epsilon) \epsilon$ $\mathscr{B}_{\gamma}$. To show that $u(t, x)$ is quasiperiodic, it remains to prove that $v$ depends on both variables $\left(\varphi_{1}, \varphi_{2}\right)$ independently. According to Lemma 9, $\forall\left|\widehat{q}_{1}\right|_{H_{1}} \leq \bar{R}, \widetilde{\Phi}_{\epsilon, N}\left(\widehat{q}_{1}\right) \geq \beta$. On the other hand, $\widetilde{\Phi}_{\epsilon, N}\left(\widehat{q}_{-}+\widehat{q}_{0}\right) \leq \beta / 2, \forall\left|\widehat{q}_{-}+\widehat{q}_{0}\right|_{H_{1}} \leq \bar{R}$, so that $\widehat{q}_{1} \notin Q_{-} \oplus Q_{0}$, and, therefore, $v$ depends on $\varphi_{2}$. In fact, any solution $v$ of (23) depending only on $\varphi_{2}$, that is, the solutions of

$$
\left(2 a+a^{2} \epsilon^{2}\right) \frac{d^{2} v\left(\varphi_{2}\right)}{d \varphi_{2}^{2}}+f\left(\varphi_{1}, \varphi_{2}, v\left(\varphi_{2}\right), \epsilon\right)=0,
$$

is $v\left(\varphi_{2}\right) \equiv 0$. Indeed, by the homogeneity of $f\left(\varphi_{1}, \varphi_{2}, v, \epsilon\right)$, we have

$$
\epsilon^{3} f\left(\varphi_{1}, \varphi_{2}, v, \epsilon\right)=f\left(\varphi_{1}, \varphi_{2}, \epsilon v\right)=\sum_{k=3}^{\infty} a_{k}\left(\varphi_{1}, \varphi_{2}\right)(\epsilon v)^{k}
$$

Now consider a smooth function $h\left(\varphi_{1}\right)$ with zero mean, ant it satisfies $\int_{\mathbb{T}} a_{k}\left(\varphi_{1}, \varphi_{2}\right) h\left(\varphi_{1}\right) d \varphi_{1} \neq 0$ for some $k$. Multiplying (108) by $h\left(\varphi_{1}\right)$ and integrating over $[0, \pi]$, we have

$$
\begin{aligned}
& \int_{0}^{2 \pi}\left(2 a+a^{2} \epsilon^{2}\right) \frac{d^{2} v\left(\varphi_{2}\right)}{d \varphi_{2}^{2}} h\left(\varphi_{1}\right) d \varphi_{1} \\
& \quad+\int_{0}^{2 \pi} f\left(\varphi_{1}, \varphi_{2}, v\left(\varphi_{2}\right), \epsilon\right) h\left(\varphi_{1}\right) d \varphi_{1}=0 .
\end{aligned}
$$

According to $h\left(\varphi_{1}\right)$ that has zero mean and multiplying above equation by $\epsilon^{3}$, we get

$$
\begin{aligned}
\int_{0}^{2 \pi} f\left(\varphi_{1}, \varphi_{2}, \epsilon v\left(\varphi_{2}\right)\right) h\left(\varphi_{1}\right) d \varphi_{1} \\
\quad=\sum_{k=3}^{\infty}\left(\epsilon v\left(\varphi_{2}\right)\right)^{k} \int_{0}^{2 \pi} a_{k}\left(\varphi_{1}, \varphi_{2}\right) h\left(\varphi_{1}\right) d \varphi_{1}=0 .
\end{aligned}
$$

The function $G\left(z\left(\varphi_{2}\right)\right)=\sum_{k=3}^{\infty} b_{k}\left(\varphi_{2}\right)\left(z\left(\varphi_{2}\right)\right)^{k}$, with $b_{k}=$ $\int_{0}^{2 \pi} a_{k}\left(\varphi_{1}, \varphi_{2}\right) h\left(\varphi_{1}\right) d \varphi_{1}$, is a nontrivial analytic function. Thus, the equation $G\left(\epsilon v\left(\varphi_{2}\right)\right)=0$ cannot have a sequence of zeros accumulating to zero. So, for $\epsilon$ small enough, $v\left(\varphi_{2}\right) \equiv$ 0 .

\section{Waves Traveling in Opposite Directions}

Substituting $\omega_{1}=1+\epsilon, \omega_{2}=1+a \epsilon$ into (8), we get

$$
\mathscr{L}_{a, \epsilon} v+f\left(\varphi_{1}, \varphi_{2}, v\right)=0
$$

where (see (8))

$$
\begin{aligned}
\mathscr{L}_{a, \epsilon}:= & {\left[(1+\epsilon+1) \partial_{\varphi_{1}}+(a \epsilon) \partial_{\varphi_{2}}\right] \circ\left[\epsilon \partial_{\varphi_{1}}+(2+a \epsilon) \partial_{\varphi_{2}}\right] } \\
=4 \partial_{\varphi_{1}} \partial_{\varphi_{2}}+\epsilon[ & {\left[(2+\epsilon) \partial_{\varphi_{1}}^{2}+\left(2 a+a^{2} \epsilon\right) \partial_{\varphi_{2}}^{2}\right.} \\
& \left.+2(a+1+a \epsilon) \partial_{\varphi_{1}} \partial_{\varphi_{2}}\right]=\mathscr{L}_{0}+\epsilon \mathscr{L}_{1} .
\end{aligned}
$$

We rescale (112) in order to highlight the relationship between the amplitude and the variation in frequency: $v\left(\varphi_{1}, \varphi_{2}\right) \rightarrow$ $\sqrt{|\epsilon|} v\left(\varphi_{1}, \varphi_{2}\right)$, and, for convenience, we assume $\epsilon>0$. In the following, we consider the scaled equation

$$
\mathscr{L}_{a, \epsilon} v+\epsilon f\left(\varphi_{1}, \varphi_{2}, v, \epsilon\right)=0
$$

where $f\left(\varphi_{1}, \varphi_{2}, v, \epsilon\right)=a_{3}\left(\varphi_{1}, \varphi_{2}\right) v^{3}+\sqrt{\epsilon} O\left(v^{4}\right)$. 
Equation (114) is the Euler-Lagrange equation of the Lagrange action functional $\Psi_{\epsilon} \in C^{1}\left(\mathscr{H}_{\sigma, s}, \mathbb{R}\right)$ defined by

$$
\begin{array}{rl}
\Psi_{\epsilon}(v):=\int_{\mathbb{T}^{2}} & 2\left(\partial_{\varphi_{1}} v\right)\left(\partial_{\varphi_{2}} v\right)+\frac{\epsilon(2+\epsilon)}{2}\left(\partial_{\varphi_{1}} v\right)^{2} \\
& +\frac{\epsilon\left(2 a+a^{2} \epsilon\right)}{2}\left(\partial_{\varphi_{2}} v\right)^{2} \\
& +\epsilon(a+1+a \epsilon)\left(\partial_{\varphi_{1}} v\right)\left(\partial_{\varphi_{2}} v\right)-\epsilon F\left(\varphi_{1}, v, \delta\right) \\
= & \Psi_{0}(v)+\epsilon \Psi_{1}(v, \delta),
\end{array}
$$

where $F\left(\varphi_{1}, \varphi_{2}, v, \epsilon\right):=\int_{0}^{v} f\left(\varphi_{1}, \varphi_{2}, \xi, \epsilon\right) d \xi$ and $\Psi_{0}(v):=$ $\int_{\mathbb{T}^{2}} 2\left(\partial_{\varphi_{1}} v\right)\left(\partial_{\varphi_{2}} v\right)$

$$
\begin{aligned}
\Psi_{1}(v, \delta):=\int_{\mathbb{T}^{2}} & \frac{(2+\epsilon)}{2}\left(\partial_{\varphi_{1}} v\right)^{2}+\frac{2 a+a^{2} \epsilon}{2}\left(\partial_{\varphi_{2}} v\right)^{2} \\
& +(a+1+a \epsilon)\left(\partial_{\varphi_{1}} v\right)\left(\partial_{\varphi_{2}} v\right)-F\left(\varphi_{1}, \varphi_{2}, v, \epsilon\right) .
\end{aligned}
$$

In order to find critical points of $\Psi_{\epsilon}(v)$, we use the same method as in Section 3. The operator $\mathscr{L}_{a, \epsilon}$ is diagonal defined on the Banach space $\mathscr{H}_{\sigma, s}$ under the Fourier basis $e_{l_{1}, l_{2}}=$ $e^{i l_{1} \varphi_{1}} e^{i l_{2} \varphi_{2}}$ with eigenvalue $D_{l_{1}, l_{2}}=-\left[(2+\epsilon) l_{1}+a \epsilon l_{2}\right]\left[\epsilon l_{1}+\right.$ $\left.(2+a \epsilon) l_{2}\right]$. So, we have

$$
\mathscr{L}_{a, \epsilon}[v]=\sum_{\left(l_{1}, l_{2}\right) \in \mathbb{Z}^{2}} D_{l_{1}, l_{2}} \widehat{v}_{l_{1}, l_{2}} e^{i l_{1} \varphi_{1}} e^{i l_{2} \varphi_{2}}, \quad \forall v \in \mathscr{H}_{\sigma, s} .
$$

The unperturbed functional $\Psi_{0}: \mathscr{H}_{\sigma, s} \rightarrow \mathbb{R}$ possesses an infinite-dimensional linear space $Q$ of critical points, which are the solutions of the equation

$$
\mathscr{L}_{0} q=4 \partial_{\varphi_{1}} \partial_{\varphi_{2}} q=0
$$

The space $Q$ can be written as

$$
\begin{gathered}
Q=\left\{q=\sum_{\left(l_{1}, l_{2}\right) \in \mathbb{Z}^{2}} \widehat{q}_{l_{1}, l_{2}} e^{i l_{1} \varphi_{1}} e^{i l_{2} \varphi_{2}} \in \mathscr{H}_{\sigma, s} \mid \widehat{q}_{l_{1}, l_{2}}=0,\right. \\
\text { for } \left.l_{1} l_{2} \neq 0\right\} .
\end{gathered}
$$

We split $Q$ as

$$
Q=Q_{+}+Q_{0}+Q_{-}
$$

where

$$
\begin{aligned}
Q_{+} & :=\left\{q \in Q: \widehat{q}_{l_{1}, l_{2}}=0, \text { for }\left(l_{1}, l_{2}\right) \notin \Lambda_{+}\right\} \\
& =\left\{q_{+}:=q_{+}(\varphi) \in \mathscr{H}_{\sigma, s}^{0}\right\}, \\
Q_{0} & :=\left\{q: q_{0,0} \in \mathbb{R}\right\}, \\
Q_{-} & :=\left\{q \in Q: \widehat{q}_{l_{1}, l_{2}}=0 \text {, for }\left(l_{1}, l_{2}\right) \notin \Lambda_{-}\right\} \\
& =\left\{q_{-}:=q_{-}(\varphi) \in \mathscr{H}_{\sigma, s}^{0}\right\}, \\
\Lambda_{+} & :=\left\{\left(l_{1}, l_{2}\right) \in \mathbb{Z}^{2}: l_{1}=0,\left(l_{1}, l_{2}\right) \neq(0,0)\right\}, \\
\Lambda_{-} & :=\left\{\left(l_{1}, l_{2}\right) \in \mathbb{Z}^{2}: l_{2}=0,\left(l_{1}, l_{2}\right) \neq(0,0)\right\} .
\end{aligned}
$$

We decompose the space $\mathscr{H}_{\sigma, s}=Q+P$, where

$$
\begin{gathered}
P:=\left\{p=\sum_{\left(l_{1}, l_{2}\right) \in \mathbb{Z}^{2}} \widehat{p}_{l_{1}, l_{2}} e^{i l_{1} \varphi_{1}} e^{i l_{2} \varphi_{2}} \in \mathscr{H}_{\sigma, s} \mid \widehat{p}_{l_{1}, l_{2}}=0,\right. \\
\text { for } \left.l_{1} l_{2}=0\right\} .
\end{gathered}
$$

Projecting (114) onto the closed subspaces $Q$ and $P$, setting $v=q+p \in \mathscr{H}_{\sigma, s}$ with $q \in Q$ and $p \in P$, we obtain

$$
\begin{aligned}
& \text { (Q) } \mathscr{L}_{1}[q]+\Pi_{\mathrm{Q}} f\left(\varphi_{1}, \varphi_{2}, q+p, \epsilon\right)=0, \\
& \text { (P) } \mathscr{L}_{a, \epsilon}[p]+\epsilon \Pi_{P} f\left(\varphi_{1}, \varphi_{2}, q+p, \epsilon\right)=0,
\end{aligned}
$$

where $\Pi_{\mathrm{Q}}: \mathscr{H}_{\sigma, s} \rightarrow \mathrm{Q}, \Pi_{P}: \mathscr{H}_{\sigma, s} \rightarrow P$ are the projectors, respectively, onto $Q$ and $P$; moreover, they are continuous.

In the same way, we decompose the space $Q=Q_{1}+Q_{2}$. Setting $q=q_{1}+q_{2}$ with $q_{1} \in Q_{1}$ and $q_{2} \in Q_{2}$, we finally get

$$
\begin{gathered}
\left(Q_{1}\right) \mathscr{L}_{1}\left[q_{1}\right]+\Pi_{Q_{1}}\left[f\left(\varphi_{1}, \varphi_{2}, q_{1}+q_{2}+p, \epsilon\right)\right]=0 \\
\Longleftrightarrow d \Psi_{\epsilon}(v)[h]=0, \quad \forall h \in Q_{1}, \\
\left(Q_{2}\right) \mathscr{L}_{1}\left[q_{2}\right]+\Pi_{Q_{2}}\left[f\left(\varphi_{1}, \varphi_{2}, q_{1}+q_{2}+p, \delta\right)\right]=0 \\
\Longleftrightarrow d \Psi_{\epsilon}(v)[h]=0, \quad \forall h \in Q_{2}, \\
(P) \mathscr{L}_{a, \epsilon}[p]+\epsilon \Pi_{P}\left[f\left(\varphi_{1}, \varphi_{2}, q_{1}+q_{2}+p, \epsilon\right)\right]=0 \\
\Longleftrightarrow d \Psi_{\epsilon}(v)[h]=0, \quad \forall h \in P .
\end{gathered}
$$

The operator $\mathscr{L}_{a, \epsilon}$ is diagonal in the Fourier basis $\left\{e^{i l_{1} \varphi_{1}} e^{i l_{2} \varphi_{2}},\left(l_{1}, l_{2}\right) \in \mathbb{Z}^{2}\right\}$ with eigenvalues $D_{l_{1}, l_{2}}=-\left[(2+\epsilon) l_{1}+\right.$ $\left.a \epsilon l_{2}\right]\left[\epsilon l_{1}+(2+a \epsilon) l_{2}\right]$. We first prove that $\mathscr{L}_{a, \epsilon}$ restricted to $P$ has a bounded inverse when $(a, \epsilon)$ belongs to the uncountable zero-measure set

$$
\mathscr{D}_{\gamma}:=\left\{(a, \epsilon) \in \mathbb{R}^{-} \times \mathbb{R},\left(\frac{a \epsilon}{2+\epsilon}, \frac{\epsilon}{2+a \epsilon}\right) \in \mathscr{E}_{\gamma},\right.
$$

$$
\left.\frac{1+\epsilon}{1+a \epsilon} \notin \mathbb{Q}, 1+\epsilon \neq 0,1+a \epsilon \neq 0,2+a \epsilon \neq 0\right\},
$$


where $\mathscr{E}_{\gamma}$ is a set of badly approximate numbers defined as

$$
\begin{aligned}
\mathscr{E}_{\gamma}:= & \left\{\left(\frac{a \epsilon}{2+\epsilon}, \frac{\epsilon}{2+a \epsilon}\right):=\left(\epsilon_{1}, \epsilon_{2}\right) \in\left(-\epsilon_{0}, \epsilon_{0}\right) \times\left(-\epsilon_{0}, \epsilon_{0}\right):\right. \\
& \left.\left|l_{1}+\epsilon_{1} l_{2}\right|>\frac{\gamma}{\left|l_{2}\right|},\left|l_{2}+\epsilon_{2} l_{1}\right|>\frac{\gamma}{\left|l_{1}\right|}\right\}
\end{aligned}
$$

for $\forall l_{1}, l_{2} \in \mathbb{Z} \backslash\{0\}$, and $0<\gamma<1 / 4, \epsilon_{0} \in(0,1 / 2)$.

Lemma 12. For $(a, \epsilon) \in \mathscr{D}_{\gamma}$, the eigenvalues $D_{l_{1}, l_{2}}$ of $\mathscr{L}_{a, \epsilon}$ restricted to $P$ satisfy

$$
\begin{array}{r}
\left|D_{l_{1}, l_{2}}\right|=\left|\left((2+\epsilon) l_{1}+a \epsilon l_{2}\right)\left(\epsilon l_{1}+(2+a \epsilon) l_{2}\right)\right|>\gamma \\
\forall l_{1} l_{2} \neq 0 .
\end{array}
$$

As a consequence, the operator $\mathscr{L}_{a, \epsilon}: P \rightarrow P$ has a bounded inverse $\mathscr{L}_{a, \epsilon}^{-1}$ and satisfies

$$
\left|\mathscr{L}_{a, \epsilon}^{-1}[h]\right|_{\sigma, s} \leq \frac{|h|_{\sigma, s}}{\gamma}, \quad \forall h \in P .
$$

Proof. Denote by $[x]$ the nearest integer close to $x$ and $\{x\}=$ $x-[x]$. If both $l_{1} \neq-\left[(a \epsilon /(2+\epsilon)) l_{2}\right]$ and $l_{2} \neq-\left[(\epsilon /(2+a \epsilon)) l_{1}\right]$, then we have

$$
\begin{aligned}
& \left|D_{l_{1}, l_{2}}\right| \\
& \quad=\left|(2+\epsilon)\left(l_{1}+\frac{a \epsilon}{2+\epsilon} l_{2}\right)\right| \cdot\left|(2+a \epsilon)\left(l_{2}+\frac{\epsilon}{2+a \epsilon} l_{1}\right)\right|>1 .
\end{aligned}
$$

If $l_{1}=-\left[(a \epsilon /(2+\epsilon)) l_{2}\right]$, then

$$
\begin{aligned}
\mid & D_{l_{1}, l_{2}} \mid \\
= & \left|(2+\epsilon)\left(l_{1}+\frac{a \epsilon}{2+\epsilon} l_{2}\right)\right| \\
& \cdot\left|\epsilon\left(l_{1}+\frac{a \epsilon}{2+\epsilon} l_{2}\right)+(2+a \epsilon) l_{2}-\frac{a \epsilon}{2+\epsilon} \epsilon l_{2}\right| \\
\geq & \frac{\gamma}{\left|l_{2}\right|} \cdot \mid(2+\epsilon)\left(\epsilon\left\{\frac{a \epsilon}{2+\epsilon} l_{2}\right\}+2 l_{2}\right. \\
& \left.+a \epsilon\left(1-\frac{\epsilon}{2+\epsilon}\right) l_{2}\right) \mid \geq \gamma .
\end{aligned}
$$

In the same way, if $l_{2}=-\left[(\epsilon /(2+a \epsilon)) l_{1}\right]$, then we have

$$
\begin{aligned}
& \quad\left|D_{l_{1}, l_{2}}\right| \\
& \geq \frac{\gamma}{\left|l_{1}\right|} \cdot \mid(2+a \epsilon)\left(a \epsilon\left\{\frac{\epsilon}{2+a \epsilon} l_{1}\right\}+2 l_{1}\right. \\
& \left.+\epsilon\left(1-\frac{a^{2} \epsilon}{2+a \epsilon}\right) l_{1}\right) \mid \geq \gamma .
\end{aligned}
$$

Lemma 13. The operator $\mathscr{L}_{1}: Q_{2} \rightarrow Q_{2}$ has a bounded inverse $\mathscr{L}_{1}^{-1}$ which satisfies

$$
\left|\mathscr{L}^{-1}[h]\right|_{\sigma, s} \leq \frac{|h|_{\sigma, s}}{N^{2}}
$$

Proof. $\mathscr{L}_{1}$ is diagonal in the Fourier basis of $Q: e^{i l_{1} \varphi_{1}} e^{i l_{2} \varphi_{2}}$ with $\left(l_{1}, l_{2}\right) \in \Lambda_{+} \cup\{(0,0)\} \cup \Lambda_{-}$with eigenvalues

$$
d_{l_{1}, l_{2}}= \begin{cases}-(2+\epsilon) l_{1}^{2}, & \text { if } l_{2}=0 \\ -a(2+a \epsilon) l_{2}^{2}, & \text { if } l_{1}=0\end{cases}
$$

The eigenvalues of $\mathscr{L}_{1}$ restricted to $Q_{2}(N)$ verify $\left|d_{l_{1}, l_{2}}\right| \geq$ $N^{2} / C$, where the constant $C$ depends on $(\epsilon, a)$, and (134) holds.

Similarly, the solution of $Q_{1}$-equation (124) is the EulerLagrange equation of the reduced Lagrangian action functional:

$$
\begin{aligned}
& \Phi_{\epsilon, N}: B_{2 R} \subset Q_{1} \longrightarrow \mathbb{R}, \\
& \Phi_{\epsilon, N}\left(q_{1}\right):=\Psi_{\epsilon}\left(q_{1}+q_{2}\left(q_{1}+p\left(q_{1}\right)\right)\right) .
\end{aligned}
$$

Lemma 14. $\Phi_{\epsilon, N} \in C^{1}\left(B_{2 R}, \mathbb{R}\right)$ and a critical point $q_{1} \in B_{2 R}$ of $\Phi_{\epsilon, N}$ is a solution of the bifurcation equation (124). Moreover, $\Phi_{\epsilon, N}$ can be written as

$$
\Phi_{\epsilon, N}\left(q_{1}\right)=\text { const }+\epsilon\left(\Gamma\left(q_{1}\right)+\mathfrak{R}_{\epsilon, N}\left(q_{1}\right)\right)
$$

where

$$
\begin{aligned}
\Gamma\left(q_{1}\right):=\int_{\mathbb{T}^{2}} & \frac{(2+\epsilon)}{2}\left(\partial_{\varphi_{1}} q_{1}\right)^{2} \\
& +(1+a+a \epsilon)\left(\partial_{\varphi_{1}} q_{1}\right)\left(\partial_{\varphi_{2}} q_{1}\right) \\
& +\frac{2 a+a^{2} \epsilon}{2}\left(\partial_{\varphi_{2}} q_{1}\right)^{2}-a_{3}\left(\varphi_{1}, \varphi_{2}\right) \frac{q_{1}^{4}}{4}, \\
\mathfrak{R}_{\epsilon, N}\left(q_{1}\right):=\int_{\mathbb{T}^{2}} a_{3}\left(\varphi_{1}, \varphi_{2}\right) \frac{q_{1}^{4}}{4} & -F\left(\varphi_{1}, \varphi_{2}, q_{1}+q_{2}+p, \epsilon\right) \\
& +\frac{1}{2} f\left(\varphi_{1}, \varphi_{2}, v, \epsilon\right)\left(q_{2}+p\right),
\end{aligned}
$$

and, for some positive constant $C_{2}(R) \geq C_{1}(R)$, we can get

$$
\begin{gathered}
\left|\Re_{\epsilon, N}\left(q_{1}\right)\right| \leq C_{2}(R)\left(\sqrt{\epsilon}+\epsilon \gamma^{-1}+\frac{1}{N^{2}}\right), \\
\left|\Re_{\epsilon, N}^{\prime}\left(q_{1}\right)[h]\right| \leq C_{2}(R)\left(\sqrt{\epsilon}+\epsilon \gamma^{-1}+\frac{1}{N^{2}}\right)|h|_{H^{1}}, \\
\forall h \in Q_{1} .
\end{gathered}
$$

The problem of finding nontrivial solutions of the $Q_{1}$ equation is reduced to finding nontrivial critical points of the reduced action functional $\Phi_{\epsilon, N}$ in $B_{2 R}$. By (137), this is 
equivalent to finding critical points of the rescaled functional denoted by $\widehat{\Phi}_{\epsilon, N}$ and called the reduced action functional

$$
\begin{aligned}
\widehat{\Phi}_{\epsilon, N}\left(q_{1}\right) & =\Gamma\left(q_{1}\right)+\mathfrak{R}_{\epsilon, N}\left(q_{1}\right) \\
& \equiv\left(\mathfrak{Q}\left(q_{1}\right)-\int_{\mathbb{T}^{2}} a_{3}\left(\varphi_{1}, \varphi_{2}\right) \frac{q_{1}^{4}}{4}+\mathfrak{R}_{\epsilon, N}\left(q_{1}\right)\right),
\end{aligned}
$$

where the quadratic form

$$
\begin{aligned}
\mathfrak{Q}\left(q_{1}\right)= & \int_{\mathbb{T}^{2}} \frac{(2+\epsilon)}{2}\left(\partial_{\varphi_{1}} q_{1}\right)^{2}+(1+a+a \epsilon)\left(\partial_{\varphi_{1}} q_{1}\right)\left(\partial_{\varphi_{2}} q_{1}\right) \\
& +\frac{2 a+a^{2} \epsilon}{2}\left(\partial_{\varphi_{2}} q_{1}\right)^{2}
\end{aligned}
$$

is positive definite on $Q_{+}$, negative definite on $Q_{-}$, and zero definite on $Q_{0}$. For $q_{1}=q_{+}+q_{-}+q_{0} \in Q_{1}$, we have

$$
\begin{aligned}
& \mathfrak{Q}\left(q_{+}\right)=\int_{\mathbb{T}^{2}} \frac{(2+\epsilon)}{2}\left(\partial_{\varphi_{1}} q_{+}\right)^{2}=\frac{\alpha_{+}}{2}\left|q_{+}\right|_{H^{1}}^{2}, \\
& \mathfrak{Q}\left(q_{0}\right)=0, \\
& \mathfrak{Q}\left(q_{-}\right)=\int_{\mathbb{T}^{2}} \frac{(a+a \epsilon)}{2}\left(\partial_{\varphi_{1}} q_{-}\right)^{2}=-\frac{\alpha_{-}}{2}\left|q_{-}\right|_{H^{1}}^{2},
\end{aligned}
$$

where the positive constants $\alpha_{+}, \alpha_{-}$are bounded away from zero and independent of $\epsilon$. The following steps of finding the nontrivial solutions of the $Q_{1}$-equation are similar to Lemmas 9 and 11 in Section 3.4.

Proof of Theorem 2. We can get the solution of (8) as follows:

$$
v=\sqrt{|\epsilon|}\left[\widehat{q}_{1}+q_{2}\left(\hat{q}_{1}\right)+p\left(\widehat{q}_{1}\right)\right]=\sqrt{|\epsilon|}\left[\widehat{q}_{\epsilon}+p\left(\widehat{q}_{1}\right)\right] .
$$

According to Lemma 13, by the regularizing property of the operator $\mathscr{L}_{1}$, the solution $\widehat{q}_{\epsilon}:=\widehat{q}_{1}+q_{2}\left(\widehat{q}_{1}\right)$ of the $(Q)$-equation belongs to $\mathscr{H}_{\sigma, s+2} \cap Q$. By the $P$-equation

$$
\begin{aligned}
& \left(\epsilon(2+\epsilon) \partial_{\varphi_{1}}^{2}+\left(4+2 a \epsilon+2 \epsilon+2 a \epsilon^{2}\right) \partial_{\varphi_{1}} \partial_{\varphi_{2}}\right) \widehat{p} \\
& \quad=-\epsilon\left(\left(2 a+a^{2} \epsilon\right) \partial_{\varphi_{2}}^{2}+\Pi_{P} f\left(\varphi_{1}, \varphi_{2}, \widehat{q}_{\epsilon}+p, \epsilon\right)\right) \widehat{p}
\end{aligned}
$$

where $\widehat{p}=p\left(\widehat{q}_{1}\right)$, we can get that

$$
-\epsilon\left(\left(2 a+a^{2} \epsilon\right) \partial_{\varphi_{2}}^{2}+\Pi_{P} f\left(\varphi_{1}, \varphi_{2}, \widehat{q}_{\epsilon}+p, \epsilon\right)\right) \hat{p} \in \mathscr{H}_{\sigma^{\prime}, s},
$$

for $0<\sigma^{\prime}<\sigma$, satisfying $\left|\epsilon\left(2 a+a^{2} \epsilon\right) l_{2}^{2}\right| e^{\left|l_{2}\right| \sigma^{\prime}}<e^{\left|l_{2}\right| \sigma}$. For $(a, \epsilon) \in \mathscr{D}_{\gamma}$, the eigenvalues of operator

$$
\epsilon(2+\epsilon) \partial_{\varphi_{1}}^{2}+\left(4+2 a \epsilon+2 \epsilon+2 a \epsilon^{2}\right) \partial_{\varphi_{1}} \partial_{\varphi_{2}}
$$

restricted to $P$ satisfy

$$
\begin{array}{r}
\left|\epsilon(2+\epsilon) l_{1}^{2}+\left(4+2 a \epsilon+\epsilon+a \epsilon^{2}\right) l_{1} l_{2}\right| \geq|2+\epsilon| \frac{\gamma\left|l_{1}\right|}{\left|l_{2}\right|}, \\
\forall l_{1} \neq 0, l_{2} \neq 0,
\end{array}
$$

and, thus, we deduce that $\widehat{p} \in \mathscr{H}_{\sigma^{\prime \prime}, s+1}$, for all $0<\sigma^{\prime \prime}<\sigma^{\prime}$ (satisfying $C\left|l_{2}\right| e^{\left|l_{2}\right| \sigma^{\prime \prime}}<e^{\left|l_{2}\right| \sigma^{\prime}}$ ), and $\left|\partial_{\varphi_{1}} \widehat{p}\right|_{\sigma^{\prime \prime}, s}=O(|\varepsilon| / \gamma)$. By (144),

$$
\begin{aligned}
\epsilon(2+\epsilon) \partial_{\varphi_{1}}^{2} \widehat{p} \\
=-\left(4+2 a \epsilon+2 \epsilon+2 a \epsilon^{2}\right) \partial_{\varphi_{1}} \partial_{\varphi_{2}} \widehat{p} \\
\quad-\epsilon\left(\left(2 a+a^{2} \epsilon\right) \partial_{\varphi_{2}}^{2}+\Pi_{P} f\left(\varphi_{1}, \varphi_{2}, \widehat{q}_{\epsilon}+p, \epsilon\right)\right) \widehat{p},
\end{aligned}
$$

we get $\widehat{p} \in \mathscr{H}_{\bar{\sigma}, s+2}$, and $|\widehat{p}|_{\bar{\sigma}, s+2}=O(|\epsilon| / \gamma)$, with $0<\bar{\sigma}<\sigma^{\prime \prime}$. Thus, (19) follows with $\bar{s}=s+2,0<\bar{\sigma}<\sigma^{\prime \prime}$. By (6), $u(t, x)=$ $\sqrt{|\epsilon|} v((1+\epsilon) t-x,(1+a \epsilon) t+x)$ is the solution of $(1)$, for all $(a, \epsilon) \in \mathscr{D}_{\gamma}$. Obviously, $v$ depends on both variables $\left(\varphi_{1}, \varphi_{2}\right)$ independently. So, $u(t, x)$ is a quasiperiodic solution of (1), with frequencies $\left(\omega_{1}, \omega_{2}\right)=(1+\epsilon, 1+a \epsilon)$.

\section{Conclusion}

In this paper, for the completely resonant nonlinear wave equations, under periodic boundary conditions, we obtain the existence and regularity of quasiperiodic solutions. The forced terms we consider are quasiperiodic, and, according to the linking theorem, the bifurcation equations are solved by variational method. Moreover, the solutions depending on the the spatial and time variables are coupled and in the form of traveling waves. In [28], Yuan got the existence of quasiperiodic solutions with $n \in \mathbb{N}(n \geq 3)$ frequencies by KAM theory, in which the form of the solutions is $u(t, x)=$ $v\left(\omega_{1} t, \omega_{2} t, \ldots, \omega_{n} t, x\right)$. In the future work, we will investigate the existence of quasiperiodic solutions with the traveling wave form as $u(t, x)=v\left(\omega_{1} t+x, \omega_{2}+x, \ldots, \omega_{n} t+x\right)$.

\section{Conflict of Interests}

The authors declare that there is no conflict of interests regarding the publication of this paper.

\section{Acknowledgments}

The authors express their sincere thanks to Professor Yong Li for his instructions and many invaluable suggestions. The first author was partially supported by NSFC Grant (nos. 11001042, 11171056, 11171130, and 11271062) and SRFDP Grant (no. 20100043120001). The second author is supported by NSFC Grant (nos. 11001041, 11201360, 11101170, 11202192, and 10926105), SRFDP Grant (no. 200802001008), and the State Scholarship Fund of the China Scholarship Council (nos. 2011662521 and 2011842509).

\section{References}

[1] J. Bourgain, "Quasi-periodic solutions of Hamiltonian perturbations of 2D linear Schrödinger equations," Annals of Mathematics, vol. 148, no. 2, pp. 363-439, 1998.

[2] J. Bourgain, "Periodic solutions of nonlinear wave equations," in Harmonic Analysis and Partial Differential Equations, Chicago Lectures in Mathematics, pp. 69-97, University of Chicago Press, Chicago, Ill, USA, 1999. 
[3] J. Bourgain, Green's Function Estimates for Lattice Schrödinger Operators and Applications, vol. 158 of Annals of Mathematics Studies, Princeton University Press, Princeton, NJ, USA, 2005.

[4] L. Chierchia and J. You, "KAM tori for 1D nonlinear wave equations with periodic boundary conditions," Communications in Mathematical Physics, vol. 211, no. 2, pp. 497-525, 2000.

[5] W. Craig and C. E. Wayne, "Newton's method and periodic solutions of nonlinear wave equations," Communications on Pure and Applied Mathematics, vol. 46, no. 11, pp. 1409-1498, 1993.

[6] Y. Gao, Y. Li, and J. Zhang, "Invariant tori of nonlinear Schrödinger equation," Journal of Differential Equations, vol. 246, no. 8, pp. 3296-3331, 2009.

[7] J. Geng and J. You, "A KAM theorem for Hamiltonian partial differential equations in higher dimensional spaces," Communications in Mathematical Physics, vol. 262, no. 2, pp. 343-372, 2006.

[8] J. Geng and X. Ren, "Lower dimensional invariant tori with prescribed frequency for nonlinear wave equation," Journal of Differential Equations, vol. 249, no. 11, pp. 2796-2821, 2010.

[9] J. Geng, "Almost periodic solutions for a class of higher dimensional Schrödinger equations," Frontiers of Mathematics in China, vol. 4, no. 3, pp. 463-482, 2009.

[10] J. Pöschel, "Quasi-periodic solutions for a nonlinear wave equation," Commentarii Mathematici Helvetici, vol. 71, no. 2, pp. 269-296, 1996.

[11] X. Yuan, "A KAM theorem with applications to partial differential equations of higher dimensions," Communications in Mathematical Physics, vol. 275, no. 1, pp. 97-137, 2007.

[12] C. E. Wayne, "Periodic and quasi-periodic solutions of nonlinear wave equations via KAM theory," Communications in Mathematical Physics, vol. 127, no. 3, pp. 479-528, 1990.

[13] V. Benci and P. Rabinowitz, "Critical point theorems for indefinite functionals," Inventiones Mathematicae, vol. 52, no. 3, pp. 241-273, 1979.

[14] H. Brézis and L. Nirenberg, "Forced vibrations for a nonlinear wave equation," Communications on Pure and Applied Mathematics, vol. 31, no. 1, pp. 1-30, 1978.

[15] H. Brézis, J.-M. Coron, and L. Nirenberg, "Free vibrations for a nonlinear wave equation and a theorem of P. Rabinowitz," Communications on Pure and Applied Mathematics, vol. 33, no. 5, pp. 667-684, 1980.

[16] E. R. Fadell and P. H. Rabinowitz, "Generalized cohomological index theories for Lie group actions with an application to bifurcation questions for Hamiltonian systems," Inventiones Mathematicae, vol. 45, no. 2, pp. 139-174, 1978.

[17] P. H. Rabinowitz, "Periodic solutions of nonlinear hyperbolic partial differential equations," Communications on Pure and Applied Mathematics, vol. 20, no. 1, pp. 145-205, 1967.

[18] P. H. Rabinowitz, "Free vibrations for a semilinear wave equation," Communications on Pure and Applied Mathematics, vol. 31, no. 1, pp. 31-68, 1978.

[19] P. H. Rabinowitz, Minimax Methods in Critical Point Theory with Applications to Differential Equations, vol. 65 of CBMS Regional Conference Series in Mathematics, American Mathematical Society, Providence, RI, USA, 1986.

[20] D. Bambusi and M. Berti, "A Birkhoff-Lewis-type theorem for some Hamiltonian PDEs," SIAM Journal on Mathematical Analysis, vol. 37, no. 1, pp. 83-102, 2005.

[21] D. Bambusi and S. Paleari, "Families of periodic solutions of resonant PDEs," Journal of Nonlinear Science, vol. 11, no. 1, pp. 69-87, 2001.
[22] M. Berti and P. Bolle, "Periodic solutions of nonlinear wave equations with general nonlinearities," Communications in Mathematical Physics, vol. 243, no. 2, pp. 315-328, 2003.

[23] M. Berti and P. Bolle, "Multiplicity of periodic solutions of nonlinear wave equations," Nonlinear Analysis: Theory, Methods \& Applications, vol. 56, no. 7, pp. 1011-1046, 2004.

[24] M. Berti and P. Bolle, "Cantor families of periodic solutions for completely resonant nonlinear wave equations," Duke Mathematical Journal, vol. 134, no. 2, pp. 359-419, 2006.

[25] B. V. Lidskii and E. I. Shul'man, "Periodic solutions of the equation $u_{t t}-u_{x x}+u^{3}=0$," Functional Analysis and Its Applications, vol. 22, no. 4, pp. 332-33, 1988.

[26] G. Gentile, V. Mastropietro, and M. Procesi, "Periodic solutions for completely resonant nonlinear wave equations with Dirichlet boundary conditions," Communications in Mathematical Physics, vol. 256, no. 2, pp. 437-490, 2005.

[27] G. Gentile and M. Procesi, "Conservation of resonant periodic solutions for the one-dimensional nonlinear Schrödinger equation," Communications in Mathematical Physics, vol. 262, no. 3, pp. 533-553, 2006.

[28] X. Yuan, "Quasi-periodic solutions of completely resonant nonlinear wave equations," Journal of Differential Equations, vol. 230, no. 1, pp. 213-274, 2006.

[29] M. Berti and M. Procesi, "Quasi-periodic solutions of completely resonant forced wave equations," Communications in Partial Differential Equations, vol. 31, no. 6, pp. 959-985, 2006.

[30] M. Procesi, "Quasi-periodic solutions for completely resonant non-linear wave equations in ID and 2D," Discrete and Continuous Dynamical Systems, vol. 13, no. 3, pp. 541-552, 2005.

[31] P. Baldi, "Quasi-periodic solutions of the equation $v_{t t}-v_{x x}+v^{3}=$ $f(v)$," Discrete and Continuous Dynamical Systems, vol. 15, no. 3, pp. 883-903, 2006.

[32] Y. Ma and W. Lou, "Quasi-periodic solutions of completely resonant wave equations with quasi-periodically forced vibrations," Acta Applicandae Mathematicae, vol. 112, no. 3, pp. 309-322, 2010.

[33] D. Bambusi, "Lyapunov center theorem for some nonlinear PDE's: a simple proof," Annali della Scuola Normale Superiore di Pisa. Classe di Scienze, vol. 29, no. 4, pp. 823-837, 2000.

[34] A. Ambrosetti and M. Badiale, "Homoclinics: PoincaréMelnikov type results via a variational approach," Annales de l'Institut Henri Poincare (C) Non Linear Analysis, vol. 15, no. 2, pp. 233-252, 1998.

[35] W. M. Schmidt, Diophantine Approximation, vol. 785 of Lecture Notes in Mathematics, Springer, Berlin, Germany, 1980. 


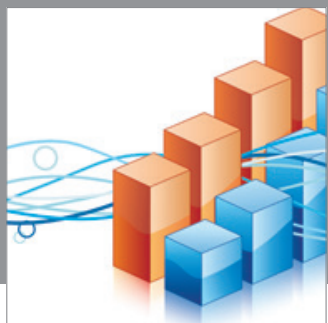

Advances in

Operations Research

mansans

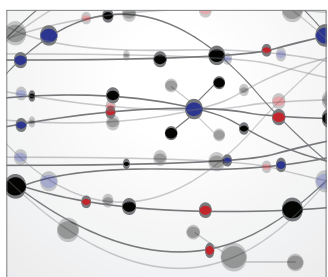

The Scientific World Journal
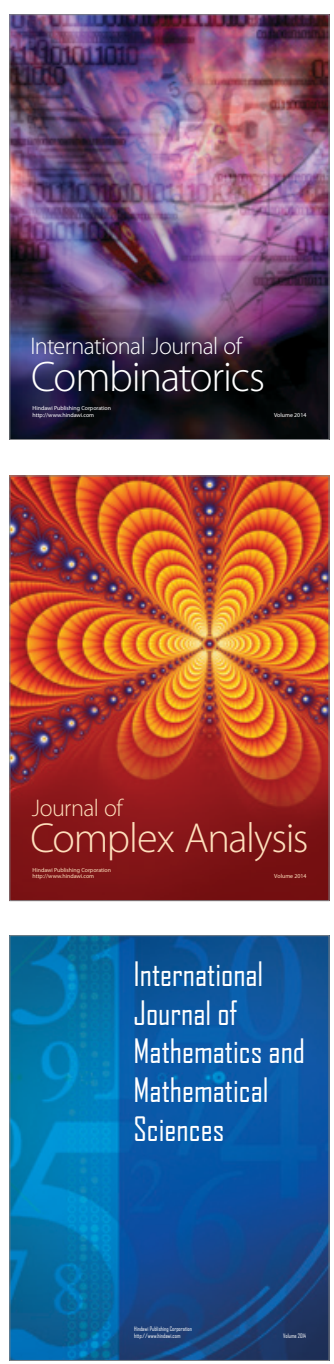
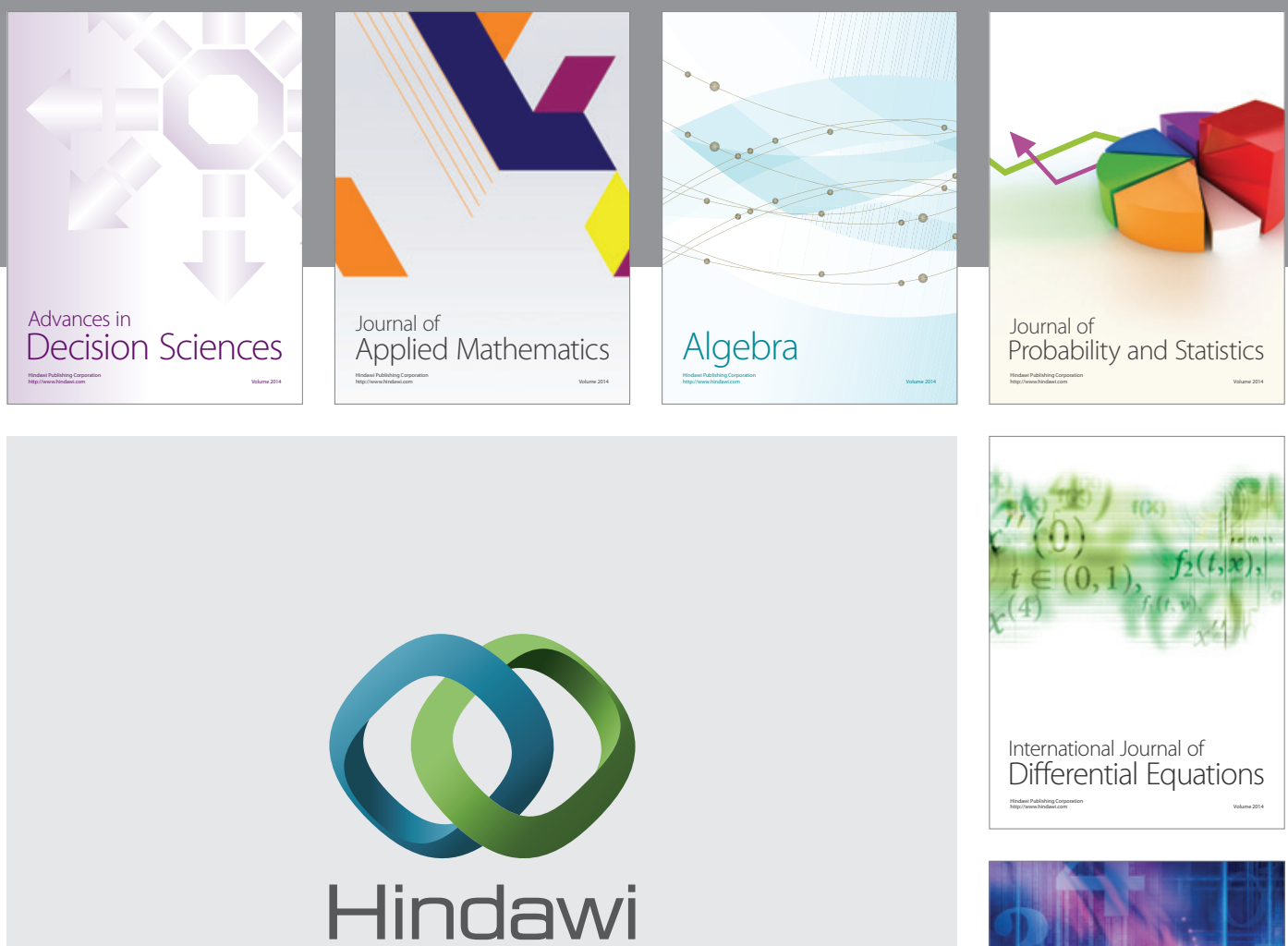

Submit your manuscripts at http://www.hindawi.com
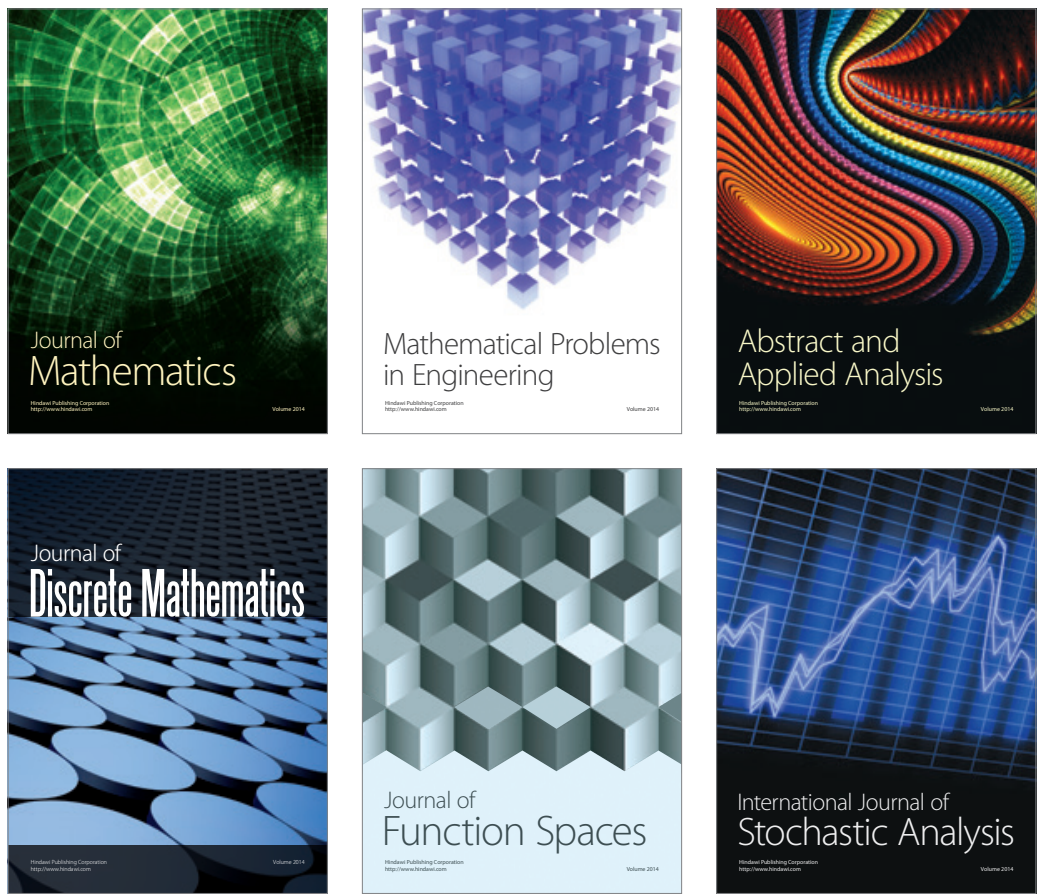

Journal of

Function Spaces

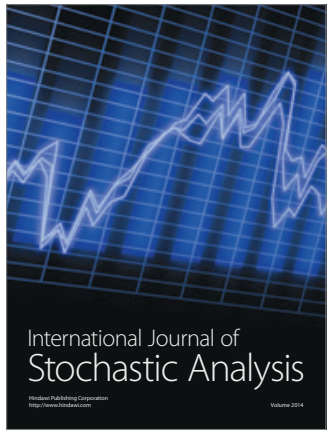

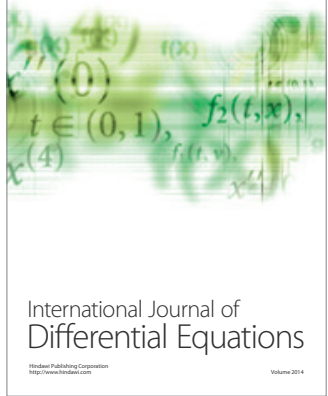
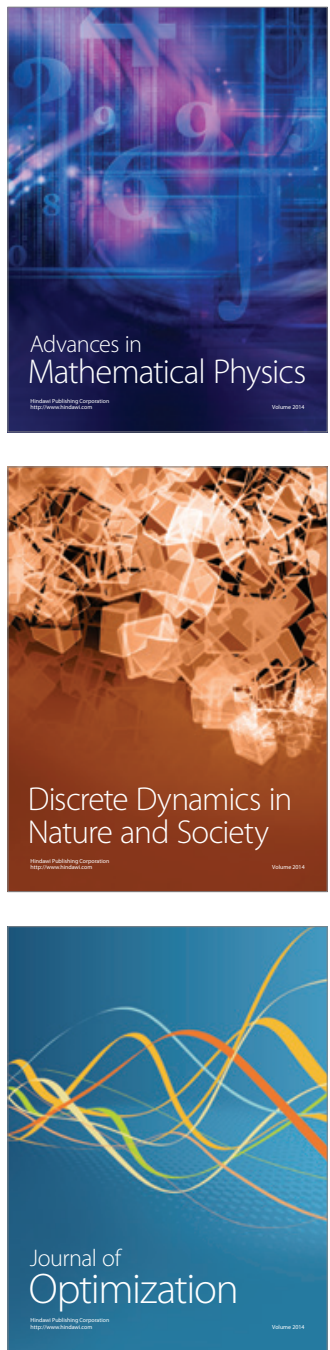